\title{
Quel(s) Aurignacien(s) à l'abri Blanchard (Sergeac, Dordogne, France) ? Données des collections d'industrie osseuse conservées aux États-Unis et retour sur le terrain
}

Which Aurignacians were at Abri Blanchard (Sergeac, Dordogne, France)? Data

from bone and antler artifacts in American collections and from new field

operations

\section{Élise Tartar, Randall White, Laurent Chiotti, Catherine Cretin et Romain} Mensan

\section{OpenEdition}

\section{Journals}

Édition électronique

URL : https://journals.openedition.org/paleo/2836

DOI : $10.4000 /$ paleo.2836

ISSN : 2101-0420

Éditeur

SAMRA

\section{Édition imprimée}

Date de publication : 28 décembre 2014

Pagination : 309-331

ISSN : 1145-3370

\section{Référence électronique}

Élise Tartar, Randall White, Laurent Chiotti, Catherine Cretin et Romain Mensan, « Quel(s)

Aurignacien(s) à l'abri Blanchard (Sergeac, Dordogne, France) ? Données des collections d'industrie osseuse conservées aux États-Unis et retour sur le terrain », PALEO [En ligne], 25 | 2014, mis en ligne le 28 juillet 2015, consulté le 21 septembre 2021. URL : http://journals.openedition.org/paleo/2836 DOI : https://doi.org/10.4000/paleo.2836 


\title{
Quel(s) Aurignacien(s) à l'abri Blanchard (Sergeac, Dordogne, France) ? Données des collections d'industrie osseuse conservées aux États-Unis et retour sur le terrain
}

\author{
Élise TARTAR ${ }^{(1)}$, Randall WHITE(2), Laurent CHIOTTI(3), \\ Catherine CRETIN(4), Romain MENSAN(5)
}

\begin{abstract}
Résumé : En 1910, M. Castanet sous la direction de L. Didon entamait les fouilles de l'abri Blanchard (Sergeac, Dordogne). Deux niveaux archéologiques séparés par une couche stérile seront individualisés et attribués à l'Aurignacien moyen sensu $\mathrm{H}$. Breuil (soit Aurignacien I à pointe à base fendue). L'abri Blanchard fait partie des deux ou trois sites ayant livré les plus riches ensembles en matières osseuses pour cette période. II recèle un important potentiel informatif pour notre connaissance des premières sociétés du Paléolithique supérieur. Mais depuis plus de 50 ans, de sérieux doutes pèsent sur l'attribution chronoculturelle faite par L. Didon, plusieurs auteurs ayant émis l'hypothèse que le niveau supérieur reflète une occupation aurignacienne plus récente. Malheureusement, les fouilles ont été réalisées selon des préoccupations et des pratiques dont découle un matériel archéologique incomplet et sans précision stratigraphique et spatiale. De plus, L. Didon rassembla le matériel des deux niveaux et le vendit par lots à des acquéreurs français et étrangers. Par conséquent, aucune étude d'ensemble n'a jamais pu être entreprise sur le matériel. Parmi ces lots, ceux envoyés aux États-Unis sont les plus méconnus. Au nombre de quatre, ils sont conservés à l'American Museum of Natural History (New York), au Wilson Museum (Castine, Maine), au Field Museum of Natural History (Chicago, Illinois) et au Logan Museum of Anthropology (Beloit, Wisconsin). Parce que l'industrie en matières osseuses y est particulièrement bien représentée, nous avons entrepris d'en mener une analyse approfondie. Cette étude nous a permis de relever la présence de matériel rapportable à l'Aurignacien ancien et récent. En outre, une récente opération de terrain a mis au jour un lambeau de couche, apportant la preuve incontestable de la présence d'Aurignacien récent dans l'abri. La position ainsi que la composition sédimentaire et archéologique du niveau permettent toutefois d'affirmer que ce dernier est en position secondaire. L'étude des collections a mis en lumière le caractère encore très lacunaire de nos connaissances sur l'Aurignacien récent. II importe de reprendre l'étude des séries et d'engager de nouvelles fouilles afin de tenter de mieux caractériser les industries et reconstituer la chronologie et l'évolution interne de l'Aurignacien du sud-ouest français.
\end{abstract}

Mots-clés : Abri Blanchard, Aurignacien ancien, Aurignacien récent, sondages, industrie en matières osseuses, pointes à base massive, grattoirs à museau, burins, lamelles Dufour sous-type Roc-de-Combe.

Abstract: Which Aurignacians were at Abri Blanchard (Sergeac, Dordogne, France)? Data from bone and antler artifacts in American collections and from new field operations. In 1910, Marcel Castanet began excavations at abri Blanchard (Sergeac, Dordogne) under the direction of Louis Didon. Two archaeological layers, separated by a sterile one, were recognized and attributed to the Middle Aurignacian (sensu Breuil), today widely known as Aurignacian I with split-based points. Even today, abri Blanchard remains one of the richest sites for this period in terms of osseous artifacts. In theory, the Blanchard assemblage retains considerable potential to contribute to our knowledge of the first societies of the Upper

(1) UMR 7041, ArScAn - Ethnologie préhistorique - elise.tartar@mae.u-paris10.fr

(2) Center for the Study of Human Origins, UMI 3199 CNRS-NYU, New York University - randall.white@nyu.edu

(3) UMR 7194, département de Préhistoire du Muséum national d'histoire naturelle - Ichiotti@mnhn.fr

(4) Centre National de Préhistoire - catherine.cretin@culture.gouv.fr

(5) UMR 5608, TRACES - mensrom@gmail.com 
Paleolithic. Nevertheless, for more than 50 years, serious doubts have been raised about L. Didon's chronocultural attribution, several authors having hypothesized that the upper level reflects a more recent Aurignacian occupation. Unfortunately, Didon and Castanet's excavations were carried out according to the concerns and practices of another time, resulting in incomplete archaeological assemblages without precise stratigraphic and spatial context. To make matters worse, L. Didon mixed the artifacts from the two levels and sold them in batches to several French and foreign buyers. This fact has made it logistically difficult to undertake a comprehensive study of this important material. Of those parts of the collection that were dispersed by L. Didon, the four sent to the United States and now curated at the American Museum of Natural History (New York), the Wilson Museum (Castine, Maine), the Field Museum of Natural History (Chicago, Illinois) and the Logan Museum of Anthropology (Beloit, Wisconsin), are the most overlooked. Osseous artifacts being well represented in these collections, we have undertaken a thorough analysis, in part to try to infer a chronocultural attribution. This study has allowed us to identify artifacts attributable to the Early and Later Aurignacian. Importantly, a recent field operation at Blanchard has led to the discovery of a fragment of a layer, providing clear evidence of Late Aurignacian in the shelter. However, the position and the sedimentary and archaeological composition of the layer suggest a derived or secondary context. Above all, the analysis of the Blanchard material has highlighted our profound lack of knowledge concerning the Late Aurignacian. It points out the necessity to prioritize the study of other older collections and undertake new excavations in an attempt to better characterize the archaeological material and to reconstruct the chronology and evolution of the Aurignacian in SW France.

Key-words: Abri Blanchard, Early Aurignacian, Late Aurignacian, test-pits, osseous industry, massive based points, nosed scrapers, burins, Dufour bladelets of Roc-de-Combe type.

\section{1 - Introduction}

L'abri Blanchard est un des nombreux gisements du vallon de Castelmerle dans la commune de Sergeac (Dordogne). Fouillé entre 1910 et 1912 par M. Castanet sous la direction de L. Didon qui y reconnut deux niveaux aurignaciens, il fait partie des deux ou trois sites ayant livré les plus riches ensembles en matières osseuses pour cette période. M. Castanet y récolta une riche industrie en silex et en matières osseuses comprenant outils et armatures, mais aussi une quantité importante de déchets de fabrication, attestant une production intense sur place. Les dépôts livrèrent également un nombre important d'éléments de parure, réalisés dans des matières diverses (coquillages atlantiques et méditerranéens, stéatite, etc.), dont l'origine témoigne de contacts ou de déplacements à très longue distance (jusqu'à $600 \mathrm{~km}$ ). S'ajoutent de nombreux objets d'art, en particulier des blocs et des plaquettes gravés. Le caractère exceptionnel du site réside aussi dans sa localisation au cœur du vallon de Castelmerle, et dans le voisinage immédiat des abris de la Souquette et de Castanet, occupés également à l'Aurignacien et ayant livré des industries et témoignages artistiques comparables. Mais depuis plus de 50 ans, de sérieux doutes pèsent sur l'attribution chronoculturelle faite par L. Didon, plusieurs auteurs ayant émis l'hypothèse que le niveau supérieur reflète une occupation aurignacienne plus récente. Malheureusement, les fouilles ont été réalisées selon des préoccupations et des pratiques dont découle un matériel archéologique incomplet et sans précision stratigraphique et spatiale. De plus, L. Didon rassembla le matériel des deux niveaux (sans indication de provenance) et le vendit par lots de taille variable à une quinzaine d'acquéreurs français et étrangers. Parmi ces lots, ceux envoyés aux États-Unis sont particulièrement méconnus. L'industrie en matières osseuses $y$ étant particulièrement bien représentée, nous avons entrepris d'en mener une analyse typo-technologique pour tenter d'en inférer une attribution chronoculturelle. Cet article propose une description détaillée des vestiges en matières osseuses, pour l'essentiel inédits. II présente également les données réunies à l'occasion d'une récente opération de terrain ayant permis la découverte de lambeaux de couches préservés.

\section{2 - L'abri Blanchard et les fouilles de L. Didon}

Débouchant sur la rive gauche de la Vézère, le vallon de Castelmerle est bordé par deux lignes de falaise dont la base forme une suite presque ininterrompue de gisements archéologiques (fig. 1). L'abri Blanchard, sur le versant est du vallon, est un abri-sous-roche aujourd'hui effondré. Situé en pied de falaise, il occupe une longue terrasse rocheuse, la même qui, à quelques mètres seulement en amont, permettra la découverte de l'abri Castanet ${ }^{6}$. L'abri de la Souquette ${ }^{7}$, occupé lui aussi à l'Aurignacien, se situe sur le versant opposé, plus au nord.

En 1909, la découverte d'une perle en surface incite M. Castanet à entreprendre un sondage. II convaint alors L. Didon de louer le terrain et d'entreprendre des fouilles

(6) L'abri Castanet est fouillé pour la première fois en 1911, par M. Castanet sous la direction de D. Peyrony (1935).

(7) L'abri de La Souquette est fouillé en 1902-1903 par l'Abbé Landesque puis par d'autres (Coste, Letellier, Hauser, Castanet) et publié pour la première fois par $F$. Delage (1938). 


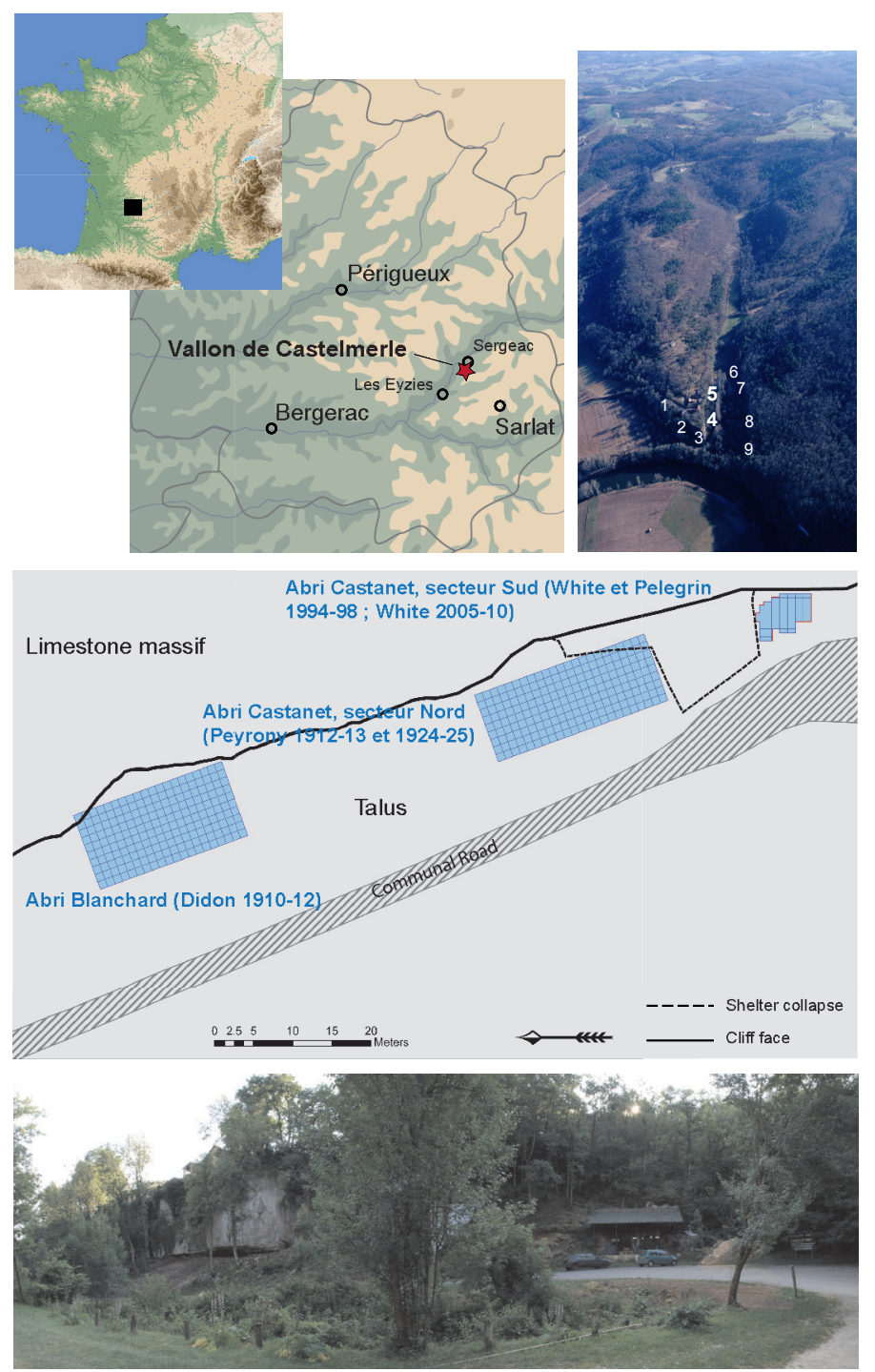

Figure 1 - Localisation du site. En haut à droite: Le vallon de Castelmerle et ses sites. 1. abri des Merveilles, 2. abri Blanchard II, 3. abri Sous-le-Roc, 4. abri Blanchard, 5. abri Castanet, 6. abri Reverdit, 7. abri du Roc d'Acier, 8. abri Labattut, 9. La Souquette. En bas: vue du versant Est du vallon.

Figure 1 - Location of the site. In the top right: The vallon de Castelmerle and its sites. 1. abri des Merveilles, 2. abri Blanchard II, 3. abri Sous-le-Roc, 4. abri Blanchard, 5. abri Castanet, 6. abri Reverdit, 7. abri du Roc d'Acier, 8. abri Labattut, 9. La Souquette. At the bottom: view of the east slope of the vallon.
(Didon 1911). Entre 1910 et 1912, M. Castanet fouille ainsi le gisement pour le compte de L. Didon. Ce dernier effectuera des visites régulières sur le site pour contrôler l'avancée des fouilles, donner des directives et récupérer le matériel mis au jour. Les deux hommes vont entretenir une correspondance presque journalière ${ }^{8}$. Deux niveaux archéologiques (B et $D$ ), séparés par une couche stérile (C), sont individualisés et attribués tous deux à l'Aurignacien à base fendue (fig. 2). C'est la raison pour laquelle L. Didon ne se donne malheureusement pas la peine d'isoler le matériel des deux niveaux : il regroupe les pièces (sans indication de provenance) et en fait la description sans jamais distinguer les deux ensembles (Didon 1911). Cependant, la coupe qu'il donne de l'abri est proche de celle que publiera D. Peyrony pour l'abri
Castanet, situé sur la même terrasse rocheuse, à quelques mètres seulement plus au sud (fig. 1), alors que D. Peyrony reconnaît un niveau de l'Aurignacien I et un niveau de l'Aurignacien II (Peyrony 1935). Les vestiges récoltés à l'abri Blanchard présentent également de fortes similitudes avec le matériel recueilli dans l'abri voisin. C'est en tout cas l'opinion de D. de Sonneville-Bordes qui note que la comparaison des graphiques cumulatifs des deux abris " confirme les ressemblances de ces outillages pratiquement identiques " (Sonneville-Bordes 1960 p. 105). C. Leroy-Prost, qui étudia l'industrie osseuse du Musée d'Archéologie Nationale, du Musée de l'Homme et de l'Institut de Paléontologie Humaine note que, comme l'abri Castanet, l'abri Blanchard « témoigne de deux stades consécutifs d'occupation aurignacienne ancienne et

(8) Cette correspondance, qui fait aujourd'hui partie des archives Didon, comprend des plans de M. Castanet indiquant des limites de couches ou la position des vestiges découverts. II n'était pas courant, à l'époque, de tenir un "carnet de fouille " mais ces lettres en font incontestablement office. 


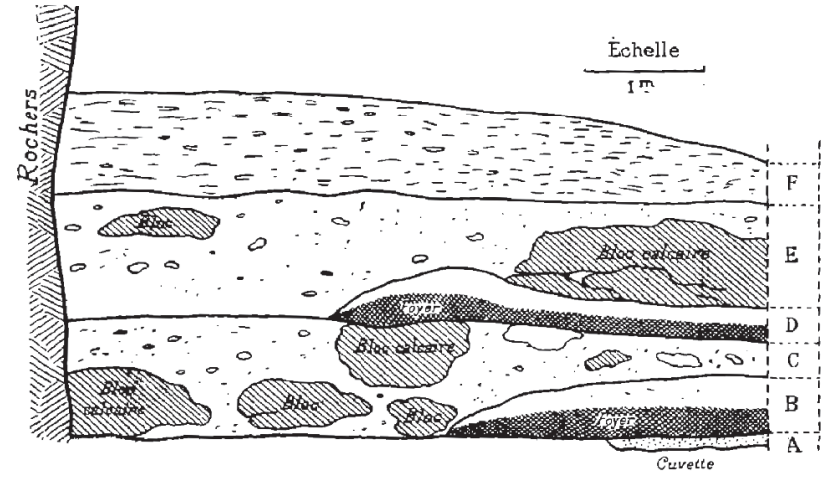

Figure 2 - Coupe stratigraphique de l'abri Blanchard publié par L. Didon (d'après figure p. 250, Didon 1911)

Figure 2 - The abri Blanchard stratigraphy as presented in Didon 1911 (after figure p. 250, Didon 1911)

moyenne » (Leroy-Prost 1979 - p. 351). Selon B. et G. Delluc "ll est vraisemblable que, si les deux gisements avaient été étudiés par un même auteur [...] ils auraient été publiés comme un ensemble » (Delluc et Delluc 1978 - p. 221).

\section{3 - Les collections Blanchard : un matériel archéologique dispersé}

Dès 1910, L. Didon vendit le produit de ses fouilles par lots de taille variable à des acquéreurs français et étrangers (White 2006a). Comme l'expliquent B. et G. Delluc, c'était une pratique courante et même encouragée à l'époque : "Dès 1910 et à chaque fois qu'on lui demande, L. DIDON vend des petites collections d'importance inégale selon les possibilités financières des acquéreurs, suivant par là les coutumes de son temps. Il est d'ailleurs encouragé dans cette voie, par les préhistoriens officiels qui estiment, compte tenu des moyens financiers limités des musées de France, que c'est là le seul moyen, pour les chercheurs bénévoles, de pourvoir aux besoins matériels de leurs campagnes de fouilles, tout en conservant aux musées français la possibilité d'acquérir les objets les plus représentatifs $\{\ldots\}$ Louis Didon semble pourtant avoir recherché activement un acquéreur unique pour la collection de l'abri Blanchard $\{. .$.$\} mais ils se rend aux$ arguments de $H$. BREUIL qui lui écrit le 23 janvier 1911 : "Je crois que vous vous trompez en croyant pouvoir trouver un acquéreur de toute la collection $\{. .$.$\} À mon sens,$ il n'y a aucune utilité, à ne pas diviser cette collection et je suis certain qu'il y aurait avantage matériel sensible à la diviser. Naturellement, vous êtes maître de penser le contraire ... Quant au musée susceptible d'acquérir l'ensemble, il n'existe pas » » (Delluc et Delluc 1981 - p. 2).

Si l'on s'appuie sur le précieux inventaire réalisé par B. et G. Delluc (1981) complété des informations réunies par R. White (2006a), les produits des fouilles de l'abri Blanchard seraient dispersés dans au moins quinze endroits différents en France et à l'étranger (tabl. 1). En conséquence, le matériel n'a encore jamais fait l'objet d'un inventaire et d'une étude d'ensemble. Parmi les collections, celles envoyées aux États-Unis sont les plus méconnues. Au nombre de quatre, elles sont conservées à l'American Museum of Natural History de New York (New York), au Wilson Museum à Castine (Maine), au Field Museum of National History à Chicago (Illinois) et au Logan Museum of Anthropology à Beloit (Wisconsin). Grâce aux archives Didon ${ }^{9}$, il est possible de connaître les circonstances dans lesquelles ont été acquises ces collections.

\begin{tabular}{|c|c|c|c|c|c|c|c|}
\hline \multirow{2}{*}{ Pays } & \multirow{2}{*}{ Villes } & \multirow{2}{*}{ Institutions } & \multicolumn{5}{|c|}{ Contenu des collections } \\
\hline & & & $\mathrm{IL}$ & IMO & $P$ & $\mathrm{BO}$ & $\mathrm{F}$ \\
\hline France & \begin{tabular}{|l|} 
Sergeac \\
Périgueux \\
Paris \\
St-Germain-en-Laye \\
Chambéry \\
\end{tabular} & $\begin{array}{l}1 \text { Musée de Castelmerle } \\
2 \text { Musée du Périgord } \\
3 \text { Musée de l'Homme } \\
4 \text { Institut de Paléontologie Humaine } \\
5 \text { Musée d'Archéologie Nationale } \\
6 \text { Collection particulière } \\
\end{array}$ & $\begin{array}{l}\mathrm{x} \\
\mathrm{x} \\
\mathrm{x} \\
\mathrm{x} \\
\mathrm{x} \\
?\end{array}$ & $\begin{array}{l}\mathrm{x} \\
\mathrm{x} \\
\mathrm{x} \\
\mathrm{x} \\
\mathrm{x} \\
?\end{array}$ & $\begin{array}{l}\mathrm{x} \\
\mathrm{x} \\
\mathrm{x} \\
\mathrm{x} \\
\mathrm{x} \\
?\end{array}$ & $\begin{array}{l}\mathrm{x} \\
\mathrm{x} \\
\mathrm{x} \\
\mathrm{x} \\
?\end{array}$ & $\begin{array}{l}? \\
? \\
? \\
? \\
? \\
?\end{array}$ \\
\hline Suisse & Bâle & 7 Museum Basel & $\bar{x}$ & $\bar{x}$ & $\bar{x}$ & & \\
\hline Pologne & Wroclow & 8 Schlesisches Museum für Kunstgewerbe und altertümer & $x$ & $x$ & $\mathrm{x}$ & & \\
\hline Grande-Bretagne & \begin{tabular}{|l} 
Jersey \\
Londres \\
Cardiff
\end{tabular} & \begin{tabular}{|l|}
9 Musée de Jersey \\
10 British Museum \\
11 National Museum of Wales
\end{tabular} & $\begin{array}{l}\mathrm{x} \\
\mathrm{x} \\
?\end{array}$ & $\begin{array}{l}\mathrm{x} \\
\mathrm{x} \\
?\end{array}$ & $x$ & ? & ? \\
\hline États-Unis & $\begin{array}{l}\text { New York, NY } \\
\text { Castine, ME } \\
\text { Beloit, WI } \\
\text { Chicago, IL }\end{array}$ & $\begin{array}{l}12 \text { American Museum of Natural History } \\
13 \text { Wilson Museum } \\
14 \text { Logan Museum of Anthropology } \\
15 \text { Field Museum of Natural History }\end{array}$ & $\begin{array}{l}\mathrm{x} \\
\mathrm{x} \\
\mathrm{x} \\
\mathrm{x}\end{array}$ & $\begin{array}{l}\mathrm{x} \\
\mathrm{x} \\
\mathrm{x} \\
\mathrm{x}\end{array}$ & $\begin{array}{l}\mathrm{x} \\
\mathrm{x} \\
\mathrm{x} \\
\mathrm{x}\end{array}$ & $\begin{array}{l}\mathrm{x} \\
\mathrm{x}\end{array}$ & $x$ \\
\hline
\end{tabular}

Tableau 1 - Lieu de conservation et contenu des collections Blanchard en France et à l'étranger (IL : industrie lithique, IMO : industrie en matières osseuses, $P$ : parure, BO : blocs ornés, $F$ : faune).

Table 1 - Location and contents of Blanchard collections in France and abroad (IL: lithic industry, IMO: osseous industry, P: ornament, BO: ornamented blocks, F: fauna).

(9) L'ensemble des archives a été retranscrit par R. White. 
Des quatre musées américains, c'est l'American Museum of National History de New York (AMNH) qui le premier se porte acquéreur d'un lot de pièces de l'abri Blanchard. En juillet 1912, E. Cartailhac annonce à L. Didon la visite prochaine de G.G. Mac Curdy accompagné de H. Fairfield Osborn, alors directeur du musée (White 2006c) : « C'est avec lui surtout que nous avons à nous entendre aussi bien dans son intérêt que dans le nôtre ». Une lettre de H. Fairfield Osborn, adressée à L. Didon la même année, prouve que le directeur de l'AMNH a bien acheté un lot d'artefacts provenant de Blanchard. G.G. Mac Curdy annoncera l'acquisition de la collection deux ans plus tard dans le bulletin du Musée (Mac Curdy 1914). La collection regroupe actuellement 201 éléments d'industrie en pierre et 59 d'industrie en matières osseuses, 18 éléments de parure, 51 restes de faune et un bloc orné.

En 1916, c'est à J. Howard Wilson que L. Didon accepte d'envoyer un lot de vestiges, un riche collectionneur qui a entrepris de créer son musée d'Anthropologie à Castine (Maine), le Wilson Museum (WM). La collection, expédiée par bateau depuis Bordeaux en août de la même année, comprend 126 éléments d'industrie en pierre, 27 d'industrie en matières osseuses, 18 éléments de parure, quelques morceaux d'ocre et de brèche.

L'achat de la collection Blanchard par le Logan Museum of Anthropology (LMA) fait suite à la création d'un département d'Anthropologie au sein du Beloit College (White 2002). G. Collie, à la tête du département, envoie un de ses étudiants en Europe, A. Pond pour procéder à l'achat de diverses collections avec l'appui financier d'un riche homme d'affaire de Chicago, F.G. Logan. En septembre 1924, A. Pond obtient l'accord de L. Didon pour l'achat du fameux collier de l'abri Blanchard, composé de près de 150 éléments de parure. Un peu plus tard, A. Pond achètera également des pièces lithiques et osseuses. À l'heure actuelle, le nombre de pièces s'élève respectivement à 44 et 22.

Enfin, en vue d'une exposition sur la préhistoire européenne au Field Museum of Natural History de Chicago (FMNH), $\mathrm{H}$. Field entreprend un voyage en France et en Espagne pour procéder à l'achat de collections assisté par $\mathrm{H}$. Breuil qui lui sert de guide et d'intermédiaire. C'est à cette occasion, pendant l'été 1927, que H. Field achète à L. Didon une collection d'artefacts de l'abri Blanchard comprenant 300 éléments d'industrie en pierre, 22 en matières osseuses, 11 éléments de parure et un bloc orné.

\section{4 - L'industrie en matières osseuses conservée aux États-Unis}

\section{Description des productions}

L'industrie en matières osseuses de l'abri Blanchard conservée dans les musées américains représente un total de 130 pièces dont 10 sont illustrées dans la publication de L. Didon (tabl. 2, fig. 3, Didon 1911). Leur nombre total correspond à plus d'un tiers de l'inventaire publié par l'auteur. Précisons toutefois que les fouilles de l'abri se sont poursuivies après 1911 et que la quantité de vestiges exhumés est par conséquent bien supérieure aux chiffres publiés. À l'exception de la collection du Logan Museum of Anthropology, étudiée par R. White (White 1992), aucune collection n'avait encore fait l'objet d'une publication. Par commodité, les artefacts sont présentés par matière première (bois de cervidé, os) et par catégorie typotechnologique, indépendamment de leur lieu de conservation (pour cette information, se référer au tableau d'inventaire, tabl. 3 - en annexe).

\section{L'industrie en bois de cervidé}

\section{Les pointes à base fendue et produits associés}

Sur les 42 fragments de pointes conservés aux États-Unis, 23 possèdent une partie proximale suffisamment bien conservée pour que leur attribution aux pointes à base fendue ne fasse aucun doute (fig. 4.1 et 4.2). Elles présentent des morphologies variables : un contour losangique à globuleux, des sections subquadrangulaires à elliptiques et un profil rectiligne (sauf 2 exemplaires au profil légèrement concave-convexe). Seules 4 sont entières ou presque entières. La plus petite, conservée au $\mathrm{FMNH}$, mesure $49 \mathrm{~mm}$ de long ( $\mathrm{N}^{\circ} 18^{10}$, fig. 4.2). La perforation de la partie distale est récente comme en témoigne la photographie de la pièce dans la publication de L. Didon où aucune perforation n'est visible $\left(\mathrm{N}^{\circ} 18\right.$, fig. 3 , planche II $\mathrm{n}^{\circ} 3$ et fig. 4.2). L'exemplaire le plus grand, exposé dans les vitrines de l'AMNH, atteint les $110 \mathrm{~mm}$ de longueur $\left(\mathrm{N}^{\circ} 10\right.$, fig. 3 , planche $I I n^{\circ} 1$ ). Pour autant que l'on puisse en juger (surface externe du bois, alvéoles du tissu spongieux), toutes les pointes ont été réalisées à partir de bois de renne, de modules moyen à gros.

Sept ébauches complètent le corpus. Elles se distinguent par l'aspect inachevé de leur mise en forme. Elles possèdent une section quadrangulaire avec des arêtes vives à la jonction entre les bords et les faces alors que ces arêtes ont été adoucies sur les pointes lors d'une dernière étape de façonnage. Ce qui les rend toutefois particulièrement reconnaissables est le fait qu'elles portent encore les stigmates du procédé IFC employé pour l'aménagement de la fente basale. Nous ne décrirons pas ici en détail ce procédé qui a fait l'objet d'une publication récente (Tartar et White 2013). Notons simplement qu'il repose sur l'emploi conjoint et complémentaire des techniques d'Incision et de Flexion, envisagées par D. Peyrony (Peyrony 1928, 1935), et de la technique du Clivage, défendue par L. Henri-Martin et reprise ensuite par H. Knecht (Henri-Martin 1931 ; Knecht 1991, 1993, 1997). Une ébauche, conservée à l'AMNH, possède des lèvres aux extrémités incisées, recourbées vers l'extérieur et entre lesquelles manque de la matière $\left(\mathrm{N}^{\circ} 100\right.$, fig. $3.1, \mathrm{~N}^{\circ} 100$, fig. 3, planche II $n^{\circ} 5$ et fig. 4.5). Elle est typique du stade 3 du procédé (après incision et flexion mais avant prolongement de la fente par clivage). Les autres ébauches

(10) Pour tous les appels de figure concernant les illustrations des pièces osseuses, le premier numéro $\left(N^{\circ}\right)$ correspond au numéro de la pièce dans le tableau 3 - en annexe. 


\begin{tabular}{|l|}
\hline INDUSTRIE EN MATIERES OSSEUSES \\
\hline Objets finis \\
Pointes à base fendue \\
Pointes à base massive \\
Pointe d'Isturitz (fragment) \\
Pointes (fragments) \\
Poinçons \\
Poignards \\
Alènes \\
Aiguilles \\
Hameçons \\
Bandeaux \\
Bâtonnets \\
Tubes \\
Lissoirs (et spatules) \\
Objets à extrémité mousse \\
Outils intermédiaires (coins, ciseaux) \\
Bâtons percés \\
Objets sur meule \\
Plaque en os décorée \\
Côte décorée \\
Retouchoirs \\
Objets indéterminés \\
\hline
\end{tabular}

Produits/Restes de fabrication

Objet en cours de mise en forme

Ebauches de pointe à base fendue

Ebauche de pointe à base massive

Pièces avec traces de sciage

Pièces avec traces de rainurage

Déchets de fente (pièces à languette)

Déchets de sectionnement

Déchets de mise en forme

Base de bois avec traces d'entaillage

TOTAL

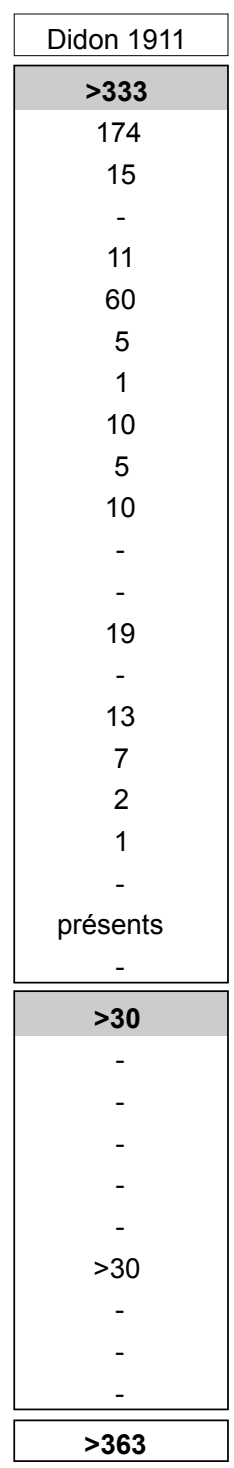

\begin{tabular}{|c|c|c|c|c|}
\hline AMNH & WM & LMA & FM & TOTAL \\
\hline $\mathbf{4 5}$ & $\mathbf{2 1}$ & $\mathbf{2 0}$ & $\mathbf{1 3}$ & $\mathbf{9 8}$ \\
15 & 3 & 2 & 3 & 23 \\
1 & 1 & - & - & 2 \\
- & - & 1 & - & 1 \\
6 & 5 & 2 & 3 & 16 \\
9 & 3 & 7 & 1 & 20 \\
- & - & - & - & - \\
- & - & - & - & - \\
- & - & - & - & - \\
- & - & - & - & - \\
- & - & - & - & - \\
- & - & 3 & - & 3 \\
- & - & 2 & - & 2 \\
6 & 9 & 1 & 1 & 17 \\
2 & - & - & 2 & 4 \\
1 & - & 1 & - & 2 \\
1 & - & - & 1 & 2 \\
- & - & - & - & - \\
- & - & - & - & - \\
1 & - & - & - & 1 \\
2 & - & - & - & 2 \\
1 & - & 1 & 1 & 3 \\
\hline 14 & $\mathbf{6}$ & $\mathbf{2}$ & $\mathbf{1 0}$ & $\mathbf{3 2}$ \\
1 & - & - & - & 1 \\
4 & 1 & - & 2 & 7 \\
1 & - & - & - & 1 \\
- & 1 & - & 3 & 4 \\
- & 1 & 1 & 1 & 3 \\
3 & 1 & 1 & 3 & 8 \\
- & 2 & - & 1 & 3 \\
4 & - & - & - & 4 \\
1 & - & - & - & 1 \\
\hline $\mathbf{5 9}$ & $\mathbf{2 7}$ & $\mathbf{2 2}$ & $\mathbf{2 2}$ & $\mathbf{1 3 0}$ \\
\hline & & & & \\
\hline \hline
\end{tabular}

Tableau 2 - Inventaire typo-technologique de l'industrie en matières osseuses de l'abri Blanchard conservée aux États-Unis.

Table 2 - Typo-technological inventory of Blanchard osseous industry housed in US museums.

appartiennent au même stade mais résultent de tentatives avortées de fente ( $\mathrm{N}^{\circ}$ 101-106). Le manque de souplesse des baguettes a provoqué la fracturation des bases lors de la flexion.

À ces pièces s'ajoutent huit pièces à languette ( $\left.\mathrm{N}^{\circ} 115-122\right)$. Comme l'avait compris $\mathrm{D}$. Peyrony, les pièces à languette sont les déchets d'aménagement des fentes produits à l'issue des opérations de sciage et flexion (Peyrony 1928, $1935)$ et non des nucléus à clavettes comme a pu le proposer H. Knecht (Knecht 1991, 1993, 1997). Certaines sont typiques, avec languette centrale. D'autres sont plus atypiques ( $\mathrm{N}^{\circ} 118$, fig. 4.2) ou résultent de tentatives de fente ratée ( $N^{\circ} 122$, fig. 4.2., Tartar et White 2013).

\section{Les pointes à base massive et produits associés}

Les collections ont livré deux pointes à base massive et une possible ébauche, toutes trois en bois de renne mais appartenant à des morpho-types distincts (Peyrony 1933). La première pointe, dont il manque l'extrémité distale, est losangique à section ovale et au profil légèrement concaveconvexe $\left(\mathrm{N}^{\circ} 25\right.$, fig. 4.6). Elle a été réalisée à partir d'un bois de gros module et son façonnage a été très soigné. La seconde pointe est un fragment mésio-proximal de pointe losangique aplatie. Elle présente une section subquadrangulaire et des dimensions nettement supérieures à la précédente ( $\mathrm{N}^{\circ} 24$, fig. 4.8). L. Didon en donne la description suivante : «... il en est une qui devait être de dimensions particulièrement importantes : la partie 

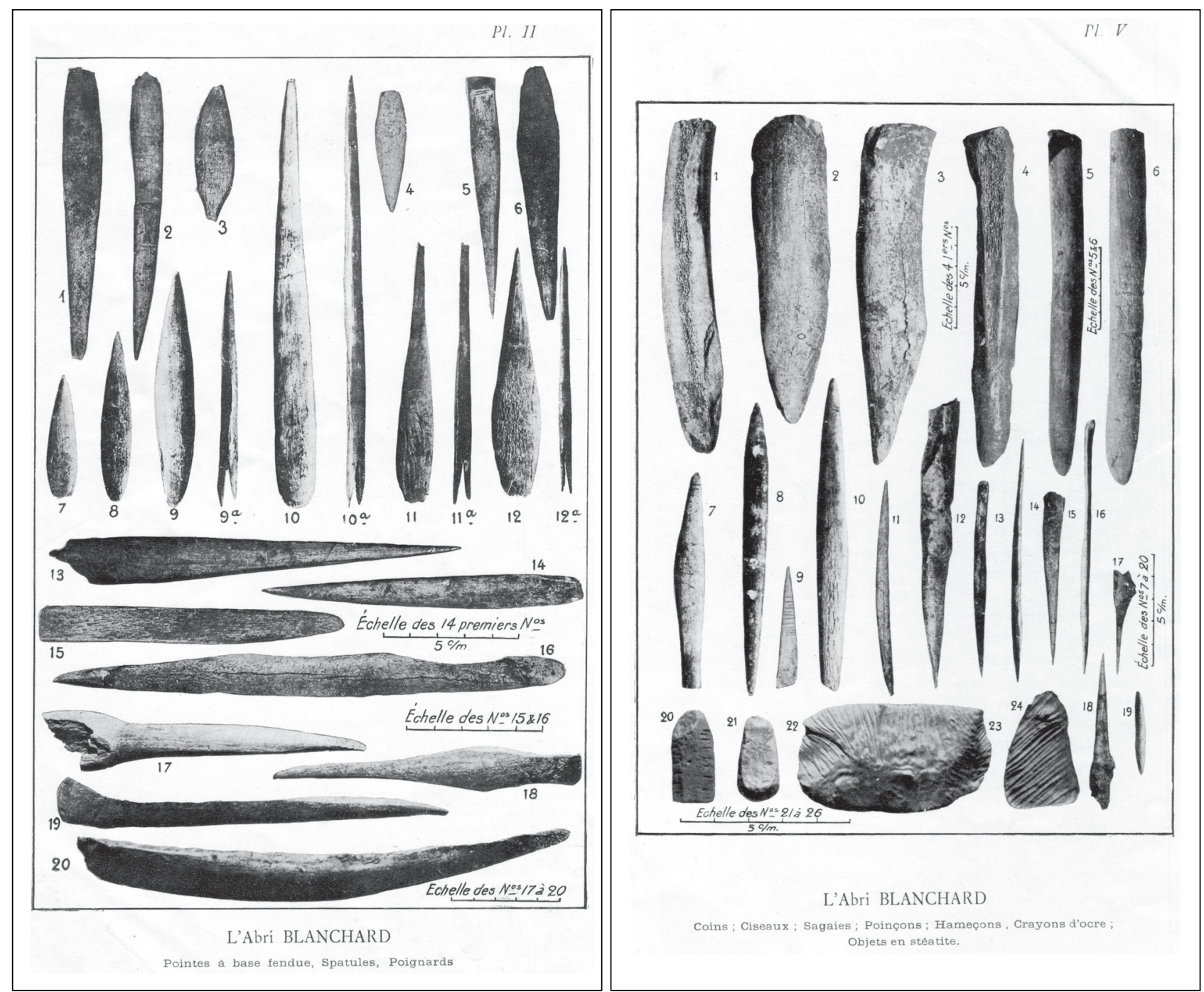

Figure 3 - Planches Il et V de la publication de L. Didon (modifié d'après Didon 1911).

Figure 3 - Plates II and V from Didon 1911 (modified after Didon 1911).

qui nous reste mesure 192 millimètres, et de sa forme on peut conclure que sa longueur était de 270 millimètres au moins. La base losangique et plate mesure 30 millimètres » (Didon 1911 - p. 258). Si cette pièce entre typologiquement dans la catégorie des pointes à base massive, la morphologie de sa base (vrillée et de section irrégulière) et ses dimensions imposantes rendent très improbable une utilisation effective en pointe de sagaie. À ces pointes, s'ajoute une probable ébauche de pointe biconique $\left(\mathrm{N}^{\circ} 107\right)$ : elle en possède la forme et son façonnage, très grossier, évoque une première étape de mise en forme.

\section{Une pointe d'Isturitz}

C'est un fragment de pièce en bois de renne aux bords convergents, de section ovalaire, couvert en face supérieure et sur les bords, de séries d'incisions légèrement obliques et parallèles entre elles $\left(\mathrm{N}^{\circ} 26\right.$, fig.
5.6). La nature, la forme et les dimensions du support ainsi que les incisions qui le recouvrent évoquent fortement la partie proximale d'une pointe d'Isturitz. Le fragment identifié au sein de la collection Blanchard du LMA offre d'ailleurs de claires similitudes avec une pointe d'Isturitz provenant du niveau 4 de l'abri Pataud (fig. 5.6 ; Movius 1973).

\section{Les fragments de pointe}

Quinze pointes n'ont pas conservé leur partie proximale ce qui empêche toute classification typologique certaine. Dans certains cas, les particularités morphométriques des fragments permettent néanmoins de les rapprocher d'un type particulier. C'est le cas de quatre fragments dont les faibles largeurs et épaisseurs et la section aplatie évoquent les pointes à base fendue $\left(\mathrm{N}^{\circ} 27,35,40,42\right)$ et de cinq fragments beaucoup plus robustes qui rappellent fortement les pointes à base massive ( $\mathrm{N}^{\circ} 30,31,34,38,39$, fig. 5.7). 


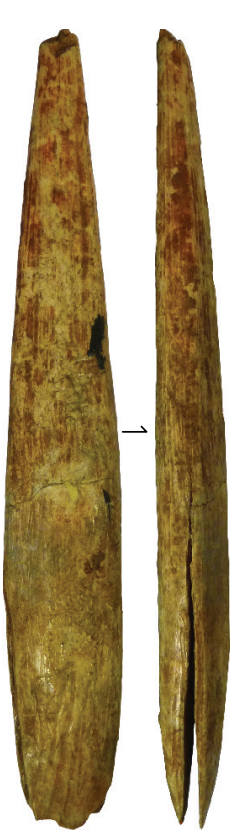

1

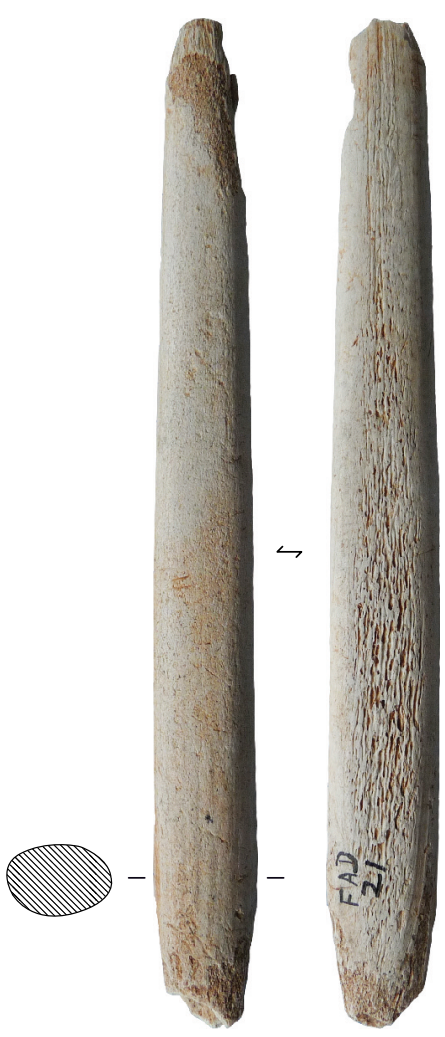

6
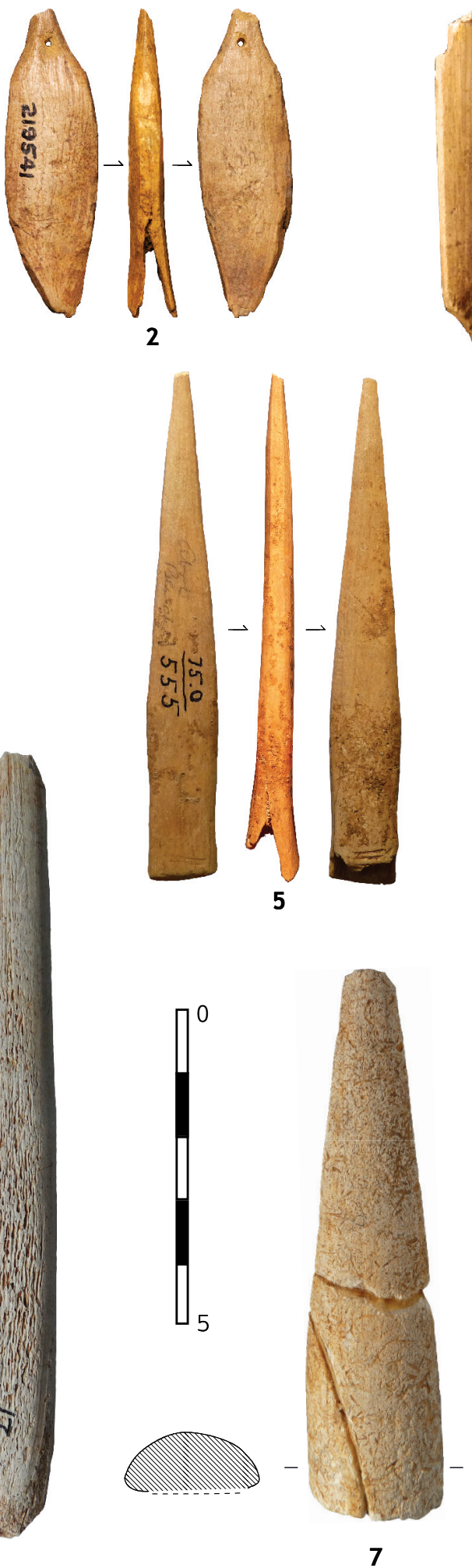
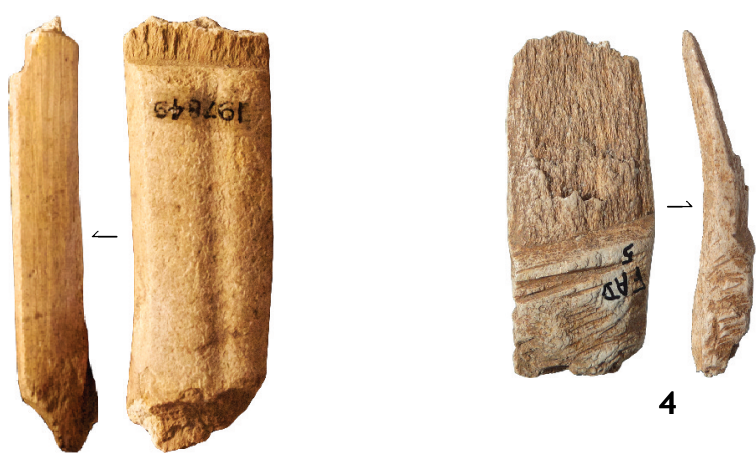

3

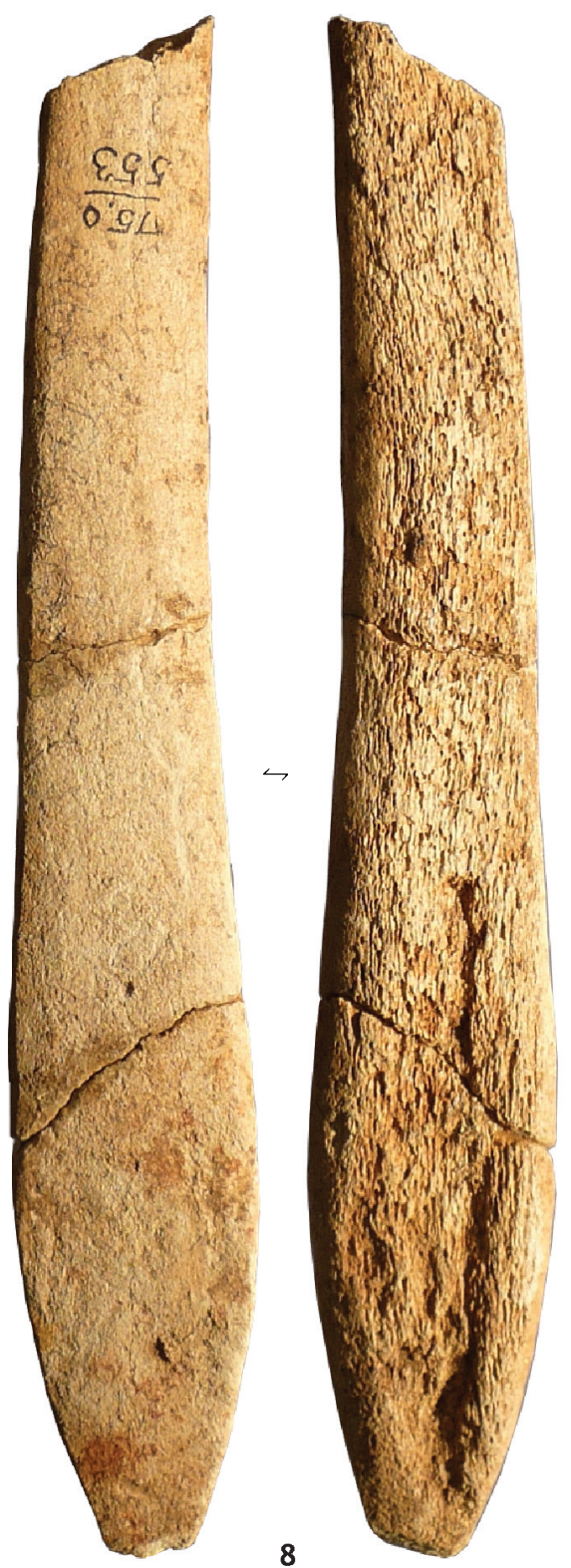

Figure 4 - 1-2: Pointes à base fendue ( $N^{\circ} 19$ et 18) ; 3-4: Pièces à languette ( $N^{\circ} 118$ et 122$) ; 5$ : Ebauche de pointe à base fendue $\left(N^{\circ} 100\right) ; 6-8$ : Pointes à base massive $\left(N^{\circ} 25,39\right.$ et 24). Coll. AMNH (5, 8) ; coll. WM (4, 6, 7) ; coll. LM (1) ; coll. FM (2-3).

Figure 4 -1-2: Split-based points ( $N^{\circ} 19$ et 18) ; 3-4: Tongued pieces ( $N^{\circ} 118$ et 122) ; 5: Split-based point rough-out ( $\left.N^{\circ} 100\right)$; 6-8: Massive-based points $\left(N^{\circ} 25,39\right.$ et 24). Coll. $A M N H(5,8)$; coll. WM $(4,6,7)$; coll. LM (1) ; coll. FM (2-3). 

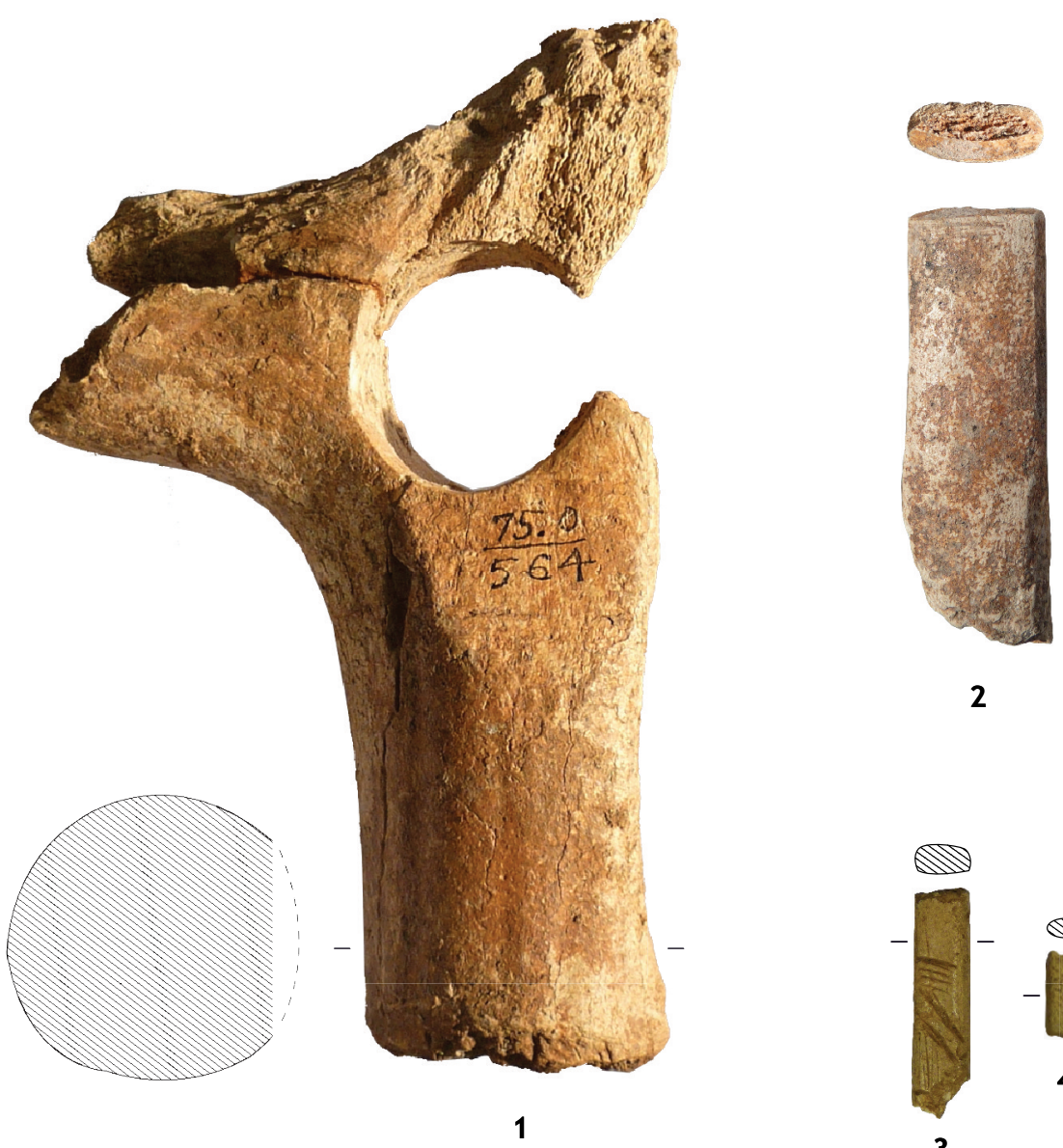

2

0 5
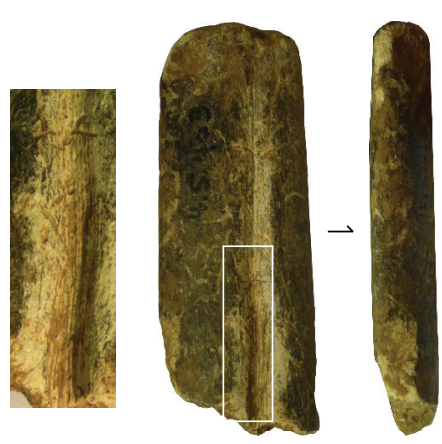

5

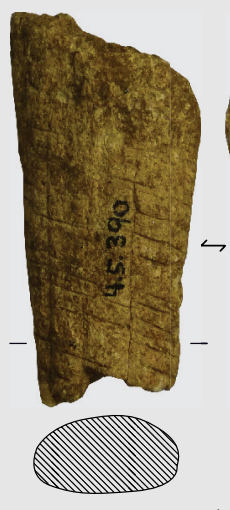

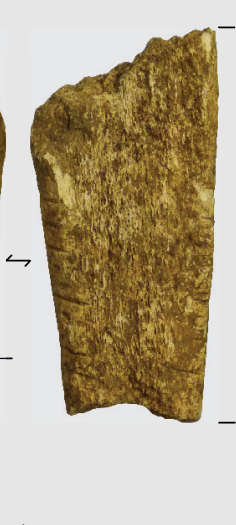

6

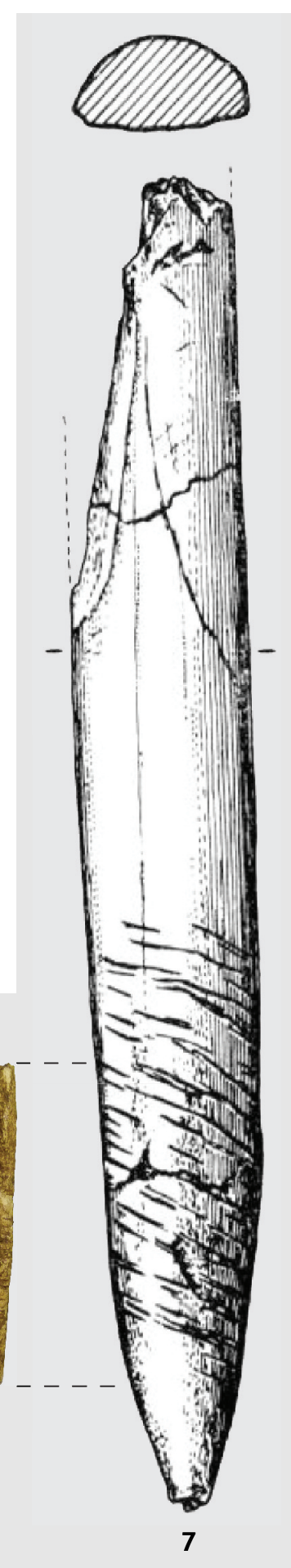

Figure 5 - 1 : Bâton percé $\left(N^{\circ} 90\right)$; 2 : Déchet de sectionnement $\left(N^{\circ} 124\right)$; 3-4 : Bâtonnets décorés $\left(N^{\circ} 63,64\right)$; 5 : Fragment d'outil intermédiaire rainuré ( $\left.N^{\circ} 113\right) ; 6$ : Fragment de pointe d'isturitz $\left(N^{\circ} 26\right) ; 7$ : Pointe d'Isturitz de l'abri Pataud, couche 4 (modifié d'après fig. 2.2, Movius 1973). Coll. AMNH (1); coll. WM (2) ; coll. LM (3-6).

Figure 5 - 1: Pierced baton ( $\left.N^{\circ} 90\right)$; 2: Waste product $\left(N^{\circ} 124\right)$; 3-4: Decorated sticks $\left(N^{\circ} 63,64\right)$; 5 : Grooved wedge fragment $\left(N^{\circ} 113\right) ; 6$ : Isturitz point fragment $\left(N^{\circ} 26\right)$; 7: Isturitz point from abri Pataud, level 4 (modified after fig. 2.2, Movius 1973). Coll. AMNH (1) ; coll. WM (2) ; coll. LM (3-6). 
La forme des fragments tend à les rapprocher du type losangique aplati pour quatre d'entre eux et du type losangique à section ovale pour le dernier.

\section{Les bâtons percés}

Il y en a deux, l'un conservé à l'AMNH et l'autre au FMNH. Pour le premier, L. Didon donne la description suivante : "Le $N^{\circ} 2$, pris dans une défense de renne beaucoup plus grosse, a le canon très court, volontairement scié. Son trou, incomplet, permet néanmoins de constater une perforation ovale, avec disparition presque totale du pas de vis. II n'y a pas d'évasement » (Didon 1911 - p. 255). Le bâton percé ( $N^{\circ}$ 90, fig. 5.1) a effectivement été réalisé sur un bois de renne de gros module, le fût de l'objet correspondant à la perche A et la branche au départ de l'andouiller d'œil ou de glace. Le second, dont il ne subsiste que la partie mésiale, provient également d'un bois de renne de gros module mais plus probablement d'une autre partie du bois, au niveau de la jonction perche C/empaumure ( $\left.\mathrm{N}^{\circ} 91\right)$.

\section{Les objets à extrémité mousse}

Comme leur nom l'indique, ces pièces ont en commun une extrémité mousse mais leur morphologie, notamment celle de l'extrémité distale, est variable. La première, illustrée dans la publication de 1911 ( $N^{\circ} 84$, fig. 3, planche $\left.V n^{\circ} 2\right)$ est un gros fragment de perche de bois de renne, de contour triangulaire. L'extrémité appointée possède un profil biseauté recouvert, en face inférieure, d'un émoussé très envahissant. Les deux objets restants ( $\mathrm{N}^{\circ} 86$ et 87 ) sont des baguettes en bois de renne brutes de débitage, obtenues par refend. Elles montrent une extrémité distale lustrée et mousse.

\section{Les bâtonnets}

II s'agit de petits fragments de baguettes en bois de renne aux bords parallèles et de section quadrangulaire ou ovalaire, mis en forme par raclage. Les pans de fracture présents aux deux extrémités témoignent d'une fracturation sur os sec (fracture récente). Ce type de pièces se rencontre fréquemment dans les ensembles du début du Paléolithique supérieur mais leur statut reste ambigu (déchets, supports, fragments d'objets ?). Deux exemplaires de l'abri Blanchard présentent un décor : l'un ponctué ( $\mathrm{N}^{\circ} 64$, fig. 5.4$)$ et l'autre composé de quelques incisions perpendiculaires et obliques ( $N^{\circ} 63$, fig. 5.3). II pourrait s'agir de fragments d'éléments de parure.

\section{Les objets indéterminés}

Le premier représente l'extrémité d'un objet façonné en bois de renne ( $\left.\mathrm{N}^{\circ} 96\right)$. Aménagé sur baguette, il possède une section très aplatie, elliptique. L'extrémité conservée présente un contour ogival et un profil rectiligne. Sa forme générale n'est pas sans rappeler celle d'un lissoir mais le mauvais état de surface empêche toute attribution typofonctionnelle. Le second est une portion mésiale d'un objet façonné sur baguette, de section ovalaire ( $\left.\mathrm{N}^{\circ} 97\right)$. Les bords sont légèrement convergents. II pourrait s'agir d'un fragment de pointe.

\section{Les produits/déchets de fabrication}

Les collections ont livré huit produits ou déchets de fabrication : trois déchets de sectionnement, deux déchets de mise en forme, deux pièces portant des traces de rainurage et une base de bois entaillée.

Les trois déchets de sectionnement sont des extrémités de baguette en bois de renne partiellement façonnées ( $\left.N^{\circ} 123-125\right)$. Ils résultent vraisemblablement d'une mise aux dimensions d'un objet au cours de sa mise en forme. Dans deux cas, le sectionnement s'est effectué par sciage ( $N^{\circ} 124$, fig. 5.2) et pour le troisième par flexion.

Les deux déchets de mise en forme résultent du façonnage d'objets fins sur baguette ( $N^{\circ} 127$ et 128). Les butées de raclage visibles au niveau de l'extrémité détachée du support en sont les stigmates caractéristiques. La forme et les dimensions des pièces évoquent les déchets produits lors de l'appointage des pointes de sagaie.

La première pièce portant des traces de rainurage est une extrémité de baguette en bois de renne de contour triangulaire dont un bord (l'autre est façonné) a conservé les stigmates d'un rainurage $\left(\mathrm{N}^{\circ} 112\right)$. La seconde est un fragment de baguette façonnée en bois de renne, sans doute l'extrémité proximale d'un outil intermédiaire de type coin ou ciseau ( $N^{\circ} 113$, fig. 5.5). En son centre, elle porte le départ d'une rainure interrompue par une fracture récente. Enfin, les restes fauniques conservés à l'AMNH ont livré une base de bois de cerf $\left(\mathrm{N}^{\circ} 130\right)$. II s'agit d'un bois de chute de mâle adulte, portant les traces d'un sectionnement par entaillage.

\section{L'industrie en os}

\section{Les poinçons}

Les collections comptent 20 poinçons de différents types : sur esquille ( $n=3, N^{\circ} 62$, fig. 6.3 ), sur portion de métapode avec partie proximale épiphysaire $\left(n=5, N^{\circ} 58\right.$, fig. 6.2), sur os naturellement effilés $(n=2)$, sur os longs entièrement façonnés ( $n=3, N^{\circ} 55$, fig. 6.1). Les autres exemplaires sont représentés par des fragments distaux et mésio-distaux $(n=7)$. À l'exception d'un exemplaire sur portion de métapode de gros module (cheval ou bovinés, $N^{\circ} 52$ ) et un autre sur os d'oiseau ou de rongeur ( $N^{\circ} 53$ ), les poinçons ont été réalisés à partir d'os de module moyen (taille renne). Notons qu'un poinçon sur esquille porte une rainure peu profonde et courbe, localisée en partie distale sur la face inférieure.

\section{Les lissoirs}

Les lissoirs comptent 16 exemplaires sur hémi-côte : un entier ( $\mathrm{N}^{\circ} 76$, fig. 6.10), deux fragments mésio-distaux, 10 fragments mésiaux et trois fragments mésio-proximaux. Les trois exemplaires ayant conservé leur partie distale 


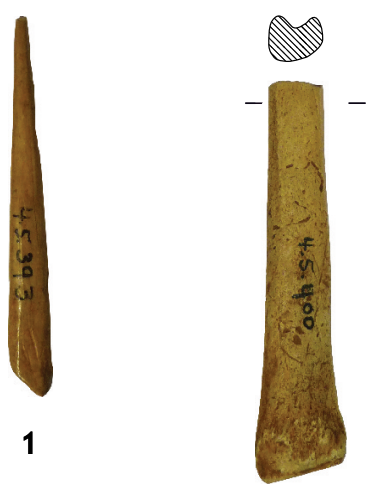

2

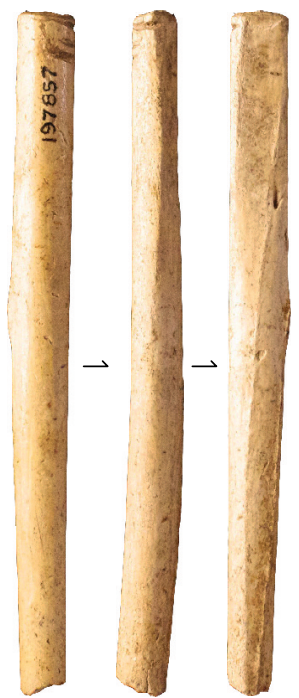

5
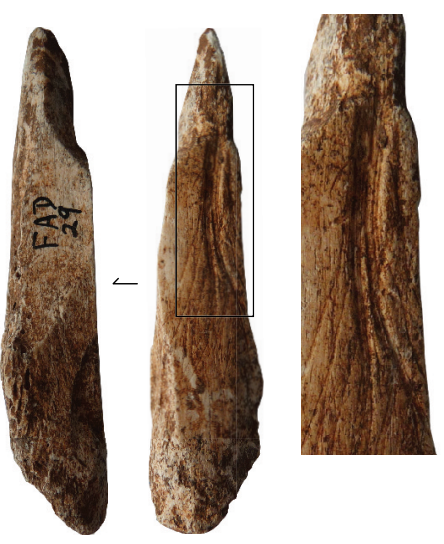

6
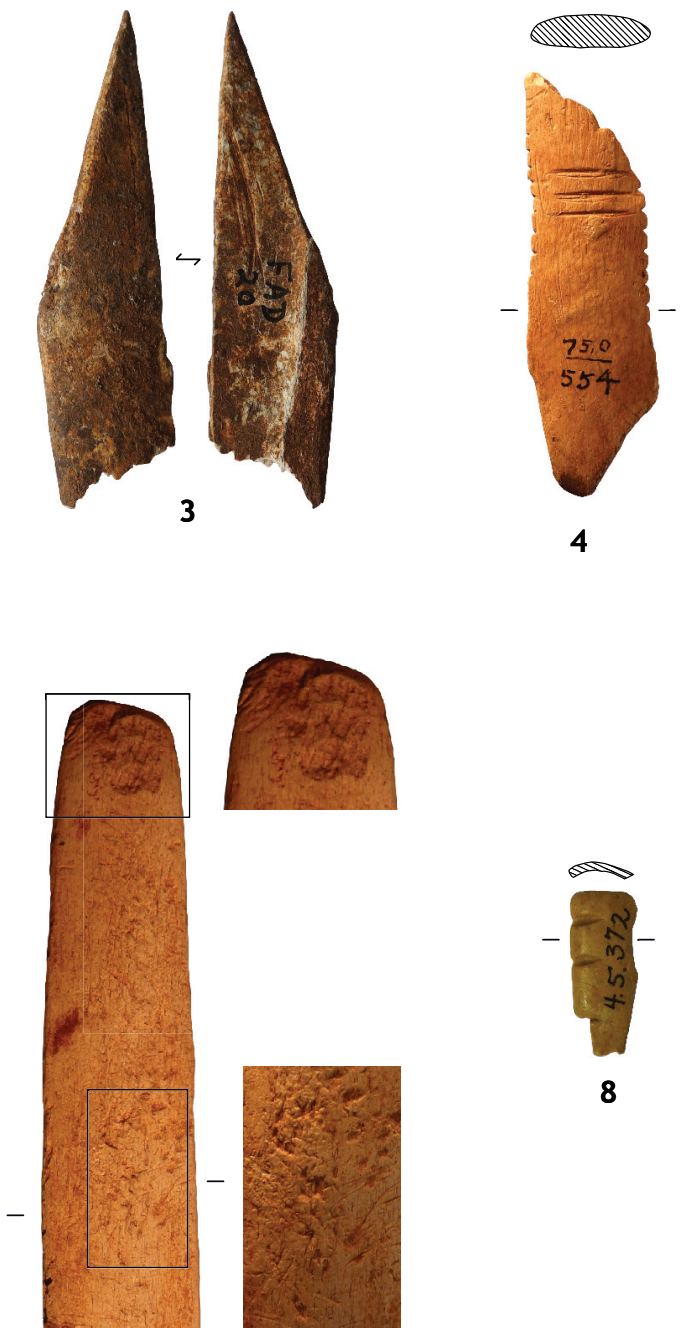

4

8

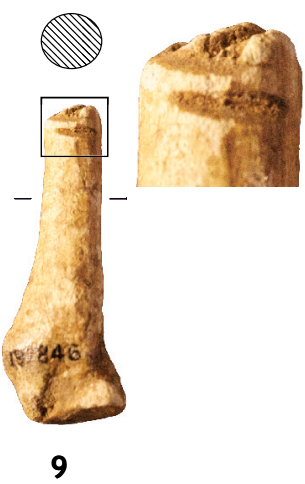

5

Figure 6 - 1-3: Poinçons $\left(N^{\circ} 55,58\right.$ et 52$) ; 4-10$ : Lissoirs $\left(N^{\circ} 71,76\right) ; 5:$ Support ou reste de fabrication de tube $\left(N^{\circ} 111\right)$; 6 : Fragment d'os rainuré ( $\left.N^{\circ} 114\right) ; 7:$ Outil intermédiaire utilisé également en retouchoir $\left(N^{\circ} 88\right) ; 8:$ Fragment de tube décoré $\left(N^{\circ} 66\right) ; 9$ : Déchet de sectionnement $\left(N^{\circ} 110\right)$. Coll. $A M N H(4,7)$; coll. WM $(3,6)$; coll. $L M(1,2,8,10)$; coll. $F M(5,9)$.

Figure 6 - 1-3: Awls (No 55, 58 et 52) ; 4-10: Lissoirs $\left(N^{\circ} 71,76\right)$; 5: Blank or waste product of tube (No 111) ; 6: Grooved bone fragment ( $\left.N^{\circ} 114\right)$; 7: Wedge used also as retoucher $\left(N^{\circ} 88\right)$; 8: Fragment of decorated tube $\left(N^{\circ} 66\right)$; 9: Bone waste product $\left(N^{\circ} 110\right)$. Coll. $A M N H(4,7)$; coll. WM $(3,6)$; coll. $\operatorname{LM}(1,2,8,10)$; coll. FM $(5,9)$. 
sont du type « lissoir commun d'axe » (Averbouh et Buisson 1996). Neuf d'entre eux portent un décor sous la forme de crans latéraux, souvent associés à des groupes d'incisions transversales en face supérieure ( $N^{\circ} 71$, fig. 6.4). Notons également que trois exemplaires portent des plages d'impressions en face supérieure qui évoquent un emploi en retouchoir ( $\mathrm{N}^{\circ} 69,70$ et 76 (fig. 6.10) ; Patou-Mathis 2002 ; Tartar 2012). La majorité d'entre eux a été réalisée sur des côtes de gros module (taille cheval/boviné).

\section{Les outils intermédiaires}

Les collections de l'AMNH et du LMA ont livré chacune un outil intermédiaire de type ciseau ( $\mathrm{N}^{\circ} 88$ et 89 ). Dans les deux cas, il s'agit d'un fragment mésio-distal d'outil réalisé sur hémi-côte. L'extrémité distale présente un fil écrasé et pour la pièce de l'AMNH, un négatif d'enlèvement et des faisceaux de stries horizontales et verticales $\left(\mathrm{N}^{\circ} 88\right.$, fig. 6.7). Comme certains lissoirs, les deux pièces portent des plages d'impressions évoquant un emploi ponctuel en retouchoir.

\section{Un objet à extrémité mousse}

II s'agit d'un fragment de scapula d'ongulé de grande taille, non façonné et dont l'extrémité, de section plate et de contour arrondi, est envahie par un important émoussé rappelant la partie active d'un lissoir ( $\mathrm{N}^{\circ} 85$ ).

\section{Les tubes}

La collection du LMA a livré deux petits fragments d'éléments tubulaires décorés de plusieurs incisions perpendiculaires à l'axe d'allongement de l'objet et parallèles entre elles ( $N^{\circ} 66$, fig. 6.8, et 67 ). Les dimensions des fragments évoquent l'emploi d'os longs d'oiseaux.

\section{Les côtes décorées}

II s'agit de deux portions de côtes décorées d'une série de crans latéraux ( $N^{\circ} 92$ et 93$)$.

\section{Les retouchoirs}

Deux retouchoirs ont été identifiés au sein des restes de faune conservés à l'AMNH ( $N^{\circ} 94$ et 95). Le premier, un fragment diaphysaire d'os long de gros module, porte une unique plage d'impressions verticales. II a été utilisé de façon prolongée comme en atteste la perte de matière au niveau de la plage. Le second est un tronçon de fémur de renne et porte une plage d'impressions verticales à chaque extrémité.

\section{Un objet indéterminé}

II s'agit d'un fragment d'objet sur baguette entièrement façonné et fracturé aux deux extrémités ( $\left.N^{\circ} 98\right)$.

\section{Les produits/déchets de fabrication}

Les produits et déchets de fabrication en os comportent : une pièce en cours de mise en forme, deux déchets de mise en forme, quatre pièces portant des traces de sciage et une portant des traces de rainurage.

La pièce en cours de mise en forme est une ébauche d'objet sur hémi-côte ( $N^{\circ} 99$ ), probablement celle d'un lissoir sinon d'un outil intermédiaire qui sont les principaux outils à avoir été réalisés sur ce type de support. La mise en forme est à peine amorcée puisque seuls les bords sont régularisés.

Quatre pièces portent à une extrémité les traces caractéristiques d'un sectionnement par sciage périphérique. Deux sont des portions de côte $\left(\mathrm{N}^{\circ} 108\right.$ et 109), vraisemblablement des déchets liés à la fabrication de lissoirs et/ou d'outils intermédiaires sur hémi-côte. La troisième est un fragment d'os long d'oiseau, à mettre en relation avec la fabrication d'éléments tubulaires (déchet ou support, $\mathrm{N}^{\circ} 111$, fig. 6.5). La dernière est une portion de métapode entièrement façonnée et ayant conservé une portion de l'extrémité épiphysaire de l'os ( $N^{\circ} 110$, fig. 6.9). À titre d'hypothèse et compte tenu du support exploité (portion de métapode) il pourrait s'agir d'un poinçon en cours de réfection, sinon d'une matrice destinée à la production de petits éléments fins de section ronde (petit élément appointé, perle ?).

Les deux déchets de mise en forme résultent, comme les exemplaires en bois de cervidé, du façonnage d'objets fins sur baguette ( $\mathrm{N}^{\circ} 126$ et 129). II s'agit probablement de déchets produits par le façonnage de parties distales de poinçons.

Enfin, un petit fragment d'os long de gros module porte en face supérieure le départ d'une rainure ( $N^{\circ} 114$, fig. 6.6). Les pans de fracture qui matérialisent les bords du fragment témoignent d'une fracturation par percussion directe diffuse de l'os à l'état frais. La rainure a donc été réalisée sur l'os long avant sa fracturation.

\section{Attribution chrono-culturelle}

$\mathrm{Au}$ sein des collections d'industrie osseuse de l'abri Blanchard conservées aux États-Unis, trois composantes typo-technologiques se distinguent. La première réunit 30 pointes à base fendue dont 7 ébauches et 8 pièces à languette associées à leur fabrication. S'y ajoutent 4 fragments de pointes dont la forme et les dimensions coïncident avec celles des pointes à base fendue. Dans sa publication, L. Didon avait déjà noté " le nombre considérable de pointes à base fendue » $(n=174)$, l'incitant à penser que l'abri Blanchard avait servi d'atelier de fabrication (Didon 1911 - p. 251). Ces pointes (et les produits associés à leur fabrication) sont également bien représentées au sein des collections françaises (LeroyProst 1979 ; Knecht 1991). Outil emblématique de l'Aurignacien (Liolios 1999, 2006 ; Tejero 2013), la pointe à base fendue sert très tôt de fossile directeur pour dater les ensembles archéologiques qui la contiennent (Leroy-Prost 1975 - p. 104), cela bien avant la classification de 
D. Peyrony qui la place comme un marqueur fort de l'Aurignacien ancien (Peyrony 1933 ; Tartar et White 2013). Parce que certains spécimens ont été identifiés en contexte protoaurignacien, l'association exclusive des pointes à base fendue à l'Aurignacien ancien fait maintenant l'objet de débats (Tartar sous presse). Pour autant, les pièces de Blanchard peuvent être raisonnablement attribuées au niveau archéologique de base de l'abri.

La seconde composante comprend 3 pointes à base massive dont une ébauche et 5 fragments dont la forme et les dimensions rappellent ce type de pointe. D'un point de vue morphométrique, ces pointes et fragments peuvent être rapprochés des morpho-types définis par D. Peyrony et érigés en fossiles directeurs des Aurignaciens II, III et IV : 5 pièces évoquent les «pointes losangiques aplaties" (Aur. II), 2 autres évoquent les «pointes losangiques à section ovale» (Aur. III) et l'ébauche évoque une « pointe biconique » (Aur. IV, Peyrony 1933). Dans sa publication, L. Didon avait déjà noté la présence de sagaies à base non fendue de différentes formes (Didon 1911 - p. 258), ce qu'a également confirmé $C$. Leroy-Prost par son étude d'une partie des collections conservées en France (Leroy-Prost 1979 - p. 326-340). La succession chronologique telle que proposée par D. Peyrony pour les pointes osseuses de l'Aurignacien récent ${ }^{11}$ n'a toutefois plus lieu d'être. Dans de nombreux sites, différents morpho-types se trouvent associés au sein des mêmes niveaux (étude en cours, E. Tartar). Sauf à envisager des mélanges systématiques, cela jette de sérieux doute sur leur succession chronologique. Une telle succession ne peut d'ailleurs avoir de valeur que si les fossiles directeurs considérés relèvent d'un même registre fonctionnel. Or, la fonction de pointe de projectile semble très discutable pour certaines pièces. C'est le cas de nombreuses pointes losangiques aplaties de La Ferrassie, des Vachons ou des Rois aux dimensions très imposantes et à l'extrémité distale mousse. La pièce $\mathrm{N}^{\circ} 24$ de l'abri Blanchard en est un bon exemple (cf. supra). Notons également qu'une part non négligeable de ces pointes ne porte pas les stigmates caractéristiques d'un emploi comme tête de projectile mais ceux d'un usage en coin, associés parfois à des plages d'impressions typiques d'un emploi en retouchoir. Un flou terminologique entoure donc la notion de pointes à base massive dont les attributs fonctionnels ne permettent pas toujours de les rattacher au registre des pointes de projectile et dont les caractéristiques morphologiques n'apparaissent pas suffisamment stables pour servir de cadre à une chronologie interne de l'Aurignacien récent. En l'état des données, leur valeur de marqueur de l'Aurignacien récent pris au sens large reste néanmoins valide. La présence de ces pointes à l'abri Blanchard vient ainsi confirmer l'existence d'Aurignacien récent dans l'abri, à rapprocher vraisemblablement du niveau archéologique supérieur décrit par L. Didon.

Une troisième composante, plus inattendue, réunit un fragment de pointe d'Isturitz et quatre pièces portant des traces de rainurage. Longtemps associées au Noaillien, les pointes d'Isturitz ont depuis été identifiées dans d'autres contextes, mais toujours gravettiens (Goutas 2008, 2013). Le rainurage est également une technique que l'on associe souvent à ce techno-complexe. C'est en effet à partir du Gravettien que la technique est intégrée au sein de procédés de débitage (comme l'extraction par double rainurage ou par rainurage bilatéral, Goutas 2009). En conclure que l'abri Blanchard a été occupé au cours du Gravettien ne se justifie pas pour autant, aucun autre vestige notamment lithique ne pouvant le confirmer. L'explication la plus plausible pour la pointe d'Isturitz est celle d'un mélange de matériel (White 1992). En 1911, tout en continuant ses recherches à l'abri Blanchard, M. Castanet entamait de nouvelles fouilles au sein du vallon de Castelmerle à l'abri Labattut, toujours pour le compte de L. Didon. II va y identifier deux niveaux gravettiens, riches en productions osseuses parmi lesquelles des pointes d'Isturitz (David 1966). Selon toute probabilité, M. Castanet ou L. Didon aura rangé par erreur le fragment de pointe de l'abri Labattut parmi le matériel de l'abri Blanchard. II n'est pas exclu que les pièces rainurées proviennent elles aussi de l'abri voisin. Cela étant et pour autant que l'on puisse en juger, trois d'entre elles ne portent qu'une simple rainure superficielle. Si l'intention recherchée nous échappe, de telles pièces ont déjà été identifiées dans des ensembles de l'Aurignacien ancien (Goutas 2009 ; Tartar 2009). La dernière pièce en revanche se distingue car la présence d'un pan de rainure sur l'un de ses bords peut attester de l'utilisation de cette technique pour délimiter le support et préparer son détachement du bloc d'origine, ce qui n'a encore jamais été signalé pour l'Aurignacien ancien. On ne peut toutefois pas exclure la possibilité que cette pièce appartienne à l'Aurignacien récent. Les modalités d'exploitation des matières osseuses pour cette période sont en effet encore très mal connues, les débats entourant l'émergence du Paléolithique supérieur en Europe ayant focalisé l'attention des chercheurs sur les industries des toutes premières phases du techno-complexe.

En définitive, l'étude des collections en matières osseuses de l'abri Blanchard conservées aux États-Unis nous a permis de relever la présence indubitable de matériel rapportable à l'Aurignacien ancien et à l'Aurignacien récent. Ces nouvelles données viennent ainsi confirmer les doutes émis depuis plus de 50 ans quant à l'équivalence chrono-

(11) Le terme "Aurignacien récent 》 est utilisé ici au sens large, tel qu'il est le plus fréquemment rencontré dans la littérature. II correspond au regroupement des stades II, III et IV de D. Peyrony (Peyrony 1933) et des phases moyenne, récente et finale de H. Delporte (Delporte 1991). Dans le cas de l'abri Blanchard, seul l'Aurignacien II de D. Peyrony (correspondant à la phase moyenne de $H$. Delporte) est présent. Cependant, nous préférons pour la suite de cet article l'emploi du terme plus générique d'« Aurignacien récent " à celui d' "Aurignacien moyen " pour désigner cette phase, d'autant plus que le terme d'Aurignacien moyen a été récemment réemployé avec une définition différente de celle de H. Delporte (Michel 2010). 
culturelle des deux niveaux archéologiques reconnus par L. Didon. Notre diagnostic a essentiellement reposé sur les pointes en bois de renne. Précisons que le reste de l'équipement, s'il est peu caractéristique d'un point de vue chrono-culturel, est néanmoins tout à fait comparable au registre de l'outillage présent au sein des ensembles aurignaciens anciens de sites voisins tels que l'abri Castanet ou l'abri Cellier. Ce constat et la supériorité numérique des pointes à base fendue par rapport aux pointes à base massive laissent envisager que la majorité de l'industrie osseuse des collections conservées aux États-Unis est originaire du niveau archéologique de base qui, par ailleurs, s'est révélé être plus épais et nettement plus riche en matériel que le niveau supérieur (Didon 1911).

\section{5 - De nouvelles données de terrain}

\section{Objectif et déroulement des opérations}

Dans le cadre des opérations de terrain entreprises en 2005 par l'équipe de R. White sur le versant est du vallon de Castelmerle, une étude topographique de ce versant a été engagée. L'objectif était de mieux apprécier la morphologie du massif calcaire et notamment de la terrasse sur laquelle se sont installés les Aurignaciens de Castanet et Blanchard. En préparation d'une étude géophysique par résistivité électrique, l'ensemble du talus a été débroussaillé laissant apparaître plusieurs tas de déblais récents et anciens. Ces différents dépôts ont été diagnostiqués et pour l'essentiel, les sondages ont atteint le substrat rocheux sans avoir livré de matériel en place, les dépôts fouillés correspondant aux déblais de M. Castanet et/ou à des remblais modernes. Cependant, une tranchée réalisée dans un tas de déblais provenant des fouilles de M. Castanet à l'abri Blanchard et situé à l'extrémité nord de sa zone de recherche, a permis la mise au jour d'un premier lambeau de couche à proximité de la paroi (secteur 1), adossé au nord contre une marche du substrat rocheux et avec un fort pendage vers le sud-ouest (fig. 7). Afin de délimiter l'étendue de ce lambeau de couche, une série de sondages a été réalisée au sud, à l'ouest puis au nord. Au nord, le secteur $4 / 5$ a livré un autre lambeau de niveau au contact du substrat rocheux, lui aussi riche en matériel archéologique mais sans lien avec le premier (la remontée du substrat rocheux séparant les deux lambeaux). La fouille de ce secteur, l'étude du matériel archéologique et une série de datations ont permis d'attribuer ce niveau à l'Aurignacien ancien et de l'envisager comme la limite nord de la couche archéologique de base fouillée par M. Castanet. Nous ne reviendrons pas sur les recherches menées dans ce secteur, l'ensemble des données étant présentées en détail dans deux articles à paraître prochainement (Bourrillon et al. sous presse ; Chiotti, Cretin, Morala sous presse), mais nous nous intéresserons au niveau mis au jour dans le secteur 1 .

\section{Analyse contextuelle du niveau archéologique du secteur 1}

Lors de sa découverte, le niveau laissait apparaître du matériel archéologique emballé dans un sédiment jaune à matrice sableuse correspondant assez bien à la description donnée par M. Castanet de son niveau archéologique supérieur. L'objectif principal des recherches a été de déterminer si ce secteur avait conservé la séquence stratigraphique décrite par L. Didon.

La fouille du secteur 1 jusqu'au substrat a révélé un niveau relativement homogène, caractérisé par un sable jaune emballant des plaquettes calcaires ayant subi une très forte carbonatation impliquant une induration du sédiment (fig. 7). Le niveau repose contre une marche formée par le substrat. Les artefacts visibles en surface sont de grosse fraction et accusent un très fort pendage sud-ouest. Leur concentration tout au long de la séquence est discontinue. Le ratio faune / élément lithique penche très nettement en faveur de ce dernier et les vestiges de faune retrouvés sont dans un état de conservation très médiocre. Sur l'ensemble des unités stratigraphiques dégagées (sept au total), la fraction décimétrique domine. Le tamisage systématique des sédiments n'a d'ailleurs révélé que très peu de fraction fine anthropique et la fouille a permis de mettre en évidence la présence d'un petit chenal. La position du niveau archéologique, sa taphonomie, la granulométrie des éléments conservés ainsi que la rareté de fraction fine anthropique nous indiquent clairement que le niveau est en position secondaire dans l'abri. Par ailleurs, la fin de la fouille de ce secteur n'a révélé aucun niveau sous-jacent. En effet, la position du dépôt en plaquage contre une marche du socle calcaire à la jonction avec la paroi de l'abri interdit toute stratigraphie à cet endroit.

La fouille du secteur 1 n'a donc pas permis de retrouver la séquence stratigraphique décrite par L. Didon. Elle a révélé la présence d'un niveau archéologique en position secondaire, accumulé par gravité et affecté par des phénomènes post-dépositionnels. Sa composition sédimentaire et l'étude du matériel archéologique qu'il contenait (cf. infra) permettent toutefois d'envisager ce niveau comme le niveau archéologique supérieur décrit par M. Castanet.

\section{Attribution chrono-culturelle du matériel archéologique du secteur 1}

Malgré sa faible surface, le secteur 1 a livré un matériel archéologique abondant largement dominé par la production lithique (1 494 objets en silex pour seulement 167 ossements.) Aucune pièce d'industrie osseuse ni de parure n'a été mise au jour dans ce secteur.

Les 1494 pièces lithiques sont composées principalement de lamelles $(44,4 \%)$ et d'éclats $(36,8 \%)$, les lames étant relativement peu nombreuses $(6,8 \%)$. Cette série comprend 103 outils, soit $7 \%$ de l'assemblage lithique (tabl. 4). Parmi ces outils, la catégorie la plus importante est de loin celle des lamelles Dufour sous-type Roc-de-Combe 
Abri Blanchard

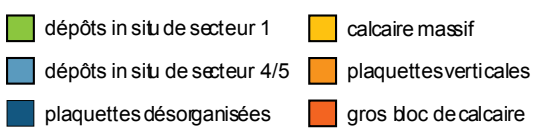

千
Fouilles 2011-2012
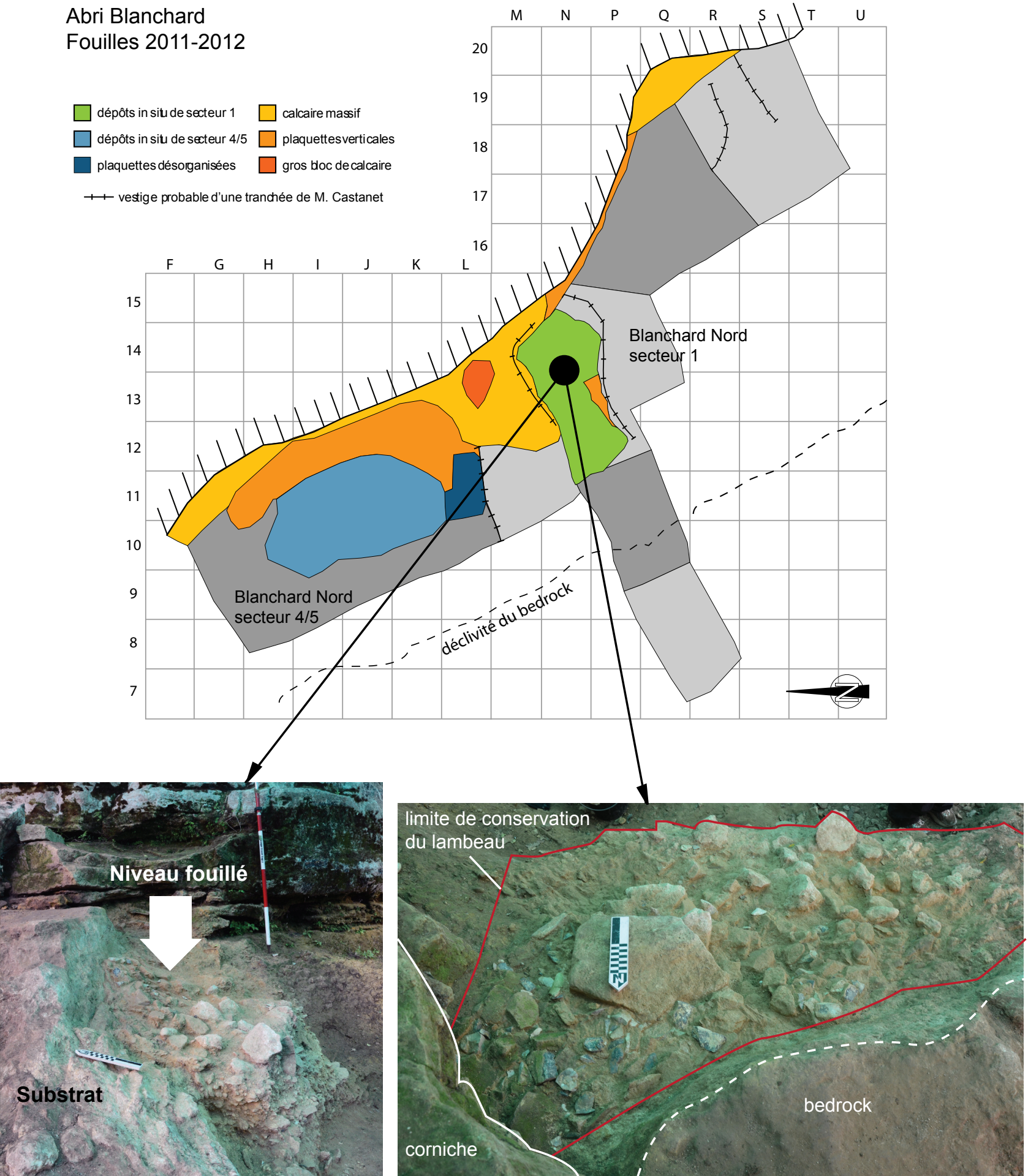

Figure 7-- l'Abri Blanchard, fouilles 2011-2012. En haut : plan de localisation des différents secteurs fouillés. En bas à gauche : vue depuis l'Ouest du lambeau de couche du secteur 1 adossé à une remontée du substrat. En bas à droite : vue supérieure du lambeau de couche du secteur 1 avant la fouille.

Figure 7 - Abri Blanchard, excavations 2011-2012. On top: plan of abri Blanchard with the different excavated sectors. Bottom left: view from the West of the sector 1 layer against the rising bedrock. Bottom right: superior view of the sector 1 layer before excavation. 
avec 33 pièces $(34,4 \%)$. L'ensemble de l'outillage sur lamelles atteint même 40,6 \%. Le groupe des grattoirs est dominé par les grattoirs épais aurignaciens : grattoirs carénés $(6,2 \%)$ et grattoirs à museau $(7,3 \%)$, présentant tous des fronts assez étroits $(17 \mathrm{~mm}$ de largeur en moyenne). Les burins sont également bien représentés, incluant quatre burins aurignaciens : trois burins carénés et un burin des Vachons. Ce sont tous des objets irréguliers, relativement atypiques. Aucun burin busqué n'est présent. Dix autres burins sont présents, dièdres, sur pan naturel, sur troncature ou sur cassure. Les pièces à retouches continues représentent $11,5 \%$ de l'outillage, mais aucune lame aurignacienne n'est attestée.

Parmi les 584 lamelles, la majorité $(76,5 \%)$, sont produites à partir de grattoirs aurignaciens (carénés ou à museau) et de burins aurignaciens (carénés, Vachons ou busqués). Pour la plupart d'entre elles (375 pièces), il est impossible de faire la différence entre production sur grattoir ou sur burin. Seules 12 pièces sont clairement issues de grattoirs aurignaciens et 121 de burins aurignaciens. La chaîne opératoire des grattoirs aurignaciens est représentée dans sa totalité, avec des mises en forme de grattoirs carénés (Chiotti et Cretin 2011), des nucléus/grattoirs carénés, des éclats de mise en forme, des éclats de ravivage, des grandes lamelles de mise en forme ou d'entretien du front et des petites lamelles de plein débitage. La production de lamelles sur des burins aurignaciens est clairement attestée par la présence de quatre burins et 121 lamelles, identifiables grâce à la présence d'un pan abrupt sur l'un de leurs bords qui correspond à une partie de la surface inférieure du support du burin (Chiotti 2003). Quelques lamelles généralement plus grandes et plus rectilignes (28 pièces, 4,2\%) sont issues d'un débitage différent : débitage dans l'épaisseur de plaquettes ou de petits blocs.

Les 33 lamelles Dufour ont une morphologie qui montre une forte sélection à l'intérieur de la production lamellaire. Elles sont de très petite taille, $16 \mathrm{~mm}$ de longueur maximale, 3,2 $\mathrm{mm}$ de largeur moyenne et $1,1 \mathrm{~mm}$ d'épaisseur moyenne, sont en majorité courbes, torses et déjetées vers la droite. Elles sont produites soit sur des grattoirs aurignaciens, soit sur des burins aurignaciens, mais aucune ne porte le pan abrupt caractéristique de ces derniers. Elles possèdent une fine retouche inverse du bord droit, le plus souvent sur toute la longueur, parfois plus intense en partie proximale. Certaines portent également une retouche du bord gauche, généralement directe. Quand leur morphologie est déterminable, les supports des huit autres lamelles retouchées ne diffèrent pas de ceux des lamelles Dufour. Leur retouche est généralement très régulière, et donc très comparable à celle des lamelles Dufour. Toutes ces pièces portent une retouche qui est nettement plus régulière et continue que celle observée sur les lamelles retouchées de l'Aurignacien ancien du site voisin de l'abri Castanet (Pelegrin et O'farell 2005 ; Chiotti, Cretin, Morala, sous presse).

L'ensemble des caractères de l'industrie lithique du secteur 1 de l'abri Blanchard se caractérise donc par la présence de : - grattoirs à museau en quantité importante ;

\begin{tabular}{|c|c|c|c|}
\hline \multicolumn{4}{|c|}{ Abri Blanchard, secteur 1, fouilles 2011-1202 } \\
\hline \multicolumn{2}{|r|}{ Type d'outil (liste type Sonneville-Bordes, Perrot) } & \multirow{2}{*}{$\begin{array}{c}\mathrm{Nb} . \\
5\end{array}$} & \multirow{2}{*}{$\begin{array}{c}\% \\
5,2\end{array}$} \\
\hline 1 & Grattoir sur bout de lame & & \\
\hline 2 & Grattoir atypique & 2 & 2,1 \\
\hline 5 & Grattoir sur lame retouchée & 2 & 2,1 \\
\hline 11 & Grattoir caréné & 5 & 5,2 \\
\hline 12 & Grattoir caréné atypique & 1 & 1,0 \\
\hline 13 & Grattoir à museau & 7 & 7,3 \\
\hline 17 & Grattoir/burin & 1 & 1,0 \\
\hline 27 & Burin dièdre droit & 1 & 1,0 \\
\hline 28 & Burin dièdre déjeté & 1 & 1,0 \\
\hline 29 & Burin dièdre d'angle & 1 & 1,0 \\
\hline 30 & Burin sur pan naturel & 2 & 2,1 \\
\hline 31 & Burin dièdre multiple & 3 & 3,1 \\
\hline 32 & Burin caréné & 2 & 2,1 \\
\hline 32 & Burin des Vachons & 1 & 1,0 \\
\hline 35 & Burin sur troncature oblique & 2 & 2,1 \\
\hline 60 & Pièce à troncature droite & 2 & 2,1 \\
\hline 61 & Pièce à troncature oblique & 1 & 1,0 \\
\hline 65 & Pièce à retouche continue sur un bord & 9 & 9,4 \\
\hline 65 & Lamelle à retouche continue sur un bord & 5 & 5,2 \\
\hline 66 & Pièce à retouche continue sur deux bords & 2 & 2,1 \\
\hline 66 & Lamelle à retouche continue sur deux bords & 1 & 1,0 \\
\hline 74 & Pièce à encoche & 3 & 3,1 \\
\hline 90 & Lamelle Dufour & 33 & 34,4 \\
\hline & Divers (fragments d'outils) & 4 & 4,2 \\
\hline \multicolumn{2}{|c|}{ Total liste type } & 96 & 100,0 \\
\hline \multicolumn{2}{|c|}{ Hors liste type Sonneville-Bordes, Perrot } & $\mathrm{Nb}$. & \\
\hline \multicolumn{2}{|c|}{ Eclat à retouche discontinue ou partielle } & 2 & \\
\hline \multicolumn{2}{|c|}{ Lame à retouche discontinue ou partielle } & 3 & \\
\hline \multicolumn{2}{|c|}{ Lamelle à retouche discontinue ou partielle } & 2 & \\
\hline \multicolumn{2}{|c|}{ Total hors liste type } & 7 & \\
\hline \multicolumn{2}{|c|}{ TOTAL OUTILLAGE } & 103 & \\
\hline
\end{tabular}

Tableau 4 - Décompte typologique de la série lithique du secteur 1 de l'abri Blanchard

Table 4 - Typological inventory of the lithic artifacts from abri Blanchard sector 1.

- grattoirs carénés généralement allongés et à fronts étroits ;

- burins de tous types ;

- burins aurignaciens (burin caréné et burin des Vachons) ;

- nombreuses lamelles issues d'une production sur burins aurignaciens ;

- petites lamelles Dufour du sous-type Roc-de-Combe en proportion très importante.

Ces différents éléments convergent vers une attribution de cette industrie à un Aurignacien récent.

\section{6 - Synthèse et conclusion}

L'étude de l'industrie en matières osseuses de l'abri Blanchard conservée aux États-Unis a permis de confirmer la présence, au sein des collections anciennes, de 
productions rapportables à l'Aurignacien récent en association avec un équipement typique de l'Aurignacien ancien. L'étude du matériel archéologique contenu dans le lambeau de couche mis au jour dans le secteur $1 \mathrm{a}$, quant à elle, apportée la preuve incontestable de la présence d'Aurignacien récent dans l'abri. La position ainsi que la composition sédimentaire et archéologique du niveau indiquent que ce dernier, accumulé par gravité et affecté par des phénomènes post-dépositionnels, est en position secondaire. Les caractéristiques de ce lambeau et les attributs typo-technologiques du matériel archéologique qu'il contenait permettent de l'envisager comme un reliquat du niveau archéologique supérieur décrit par M. Castanet. Selon toute probabilité, le caractère intrusif des dépôts du haut de la séquence n'aura pas été perçu lors des premières fouilles et L. Didon, dont la présence sur le site se limitait à des visites ponctuelles, aura publié une stratigraphie « idéalisée ».

Un constat similaire avait été effectué lors de la reprise des fouilles à l'abri Castanet (White et al. 2006b, 2007), où le rafraîchissement de la coupe formant la limite sud des fouilles de D. Peyrony en 1994 et en 2006 (Texier 2009 ; White et al. 2006b, 2007) avait permis d'identifier, en haut de la séquence et séparé de la couche archéologique de base par un niveau d'éboulis stérile, une unité comprenant du matériel archéologique assez peu caractéristique, correspondant vraisemblablement à la « couche supérieure » décrite par D. Peyrony et attribuée par ce dernier à l'Aurignacien II. La composition sédimentaire de cette unité nous avait permis de la rapporter à une phase de ruissellement intense durant laquelle des sédiments du plateau (et le matériel archéologique qu'ils contenaient) s'étaient déposés dans la partie supérieure du remplissage de l'abri.

Les abris Blanchard et Castanet ont sans doute connu une histoire archéo-stratigraphique assez proche mais les fouilles très invasives de M. Castanet, l'étendue limitée des fouilles récentes et les différents phénomènes taphonomiques qui ont affecté de façon inégale les 80 mètres de terrasse limitent fortement les interprétations possibles. Ainsi, l'hypothèse selon laquelle les deux abris auraient formé un seul et même grand site (Delluc et Delluc 1978) n'est à ce jour pas démontrée. Les archives Didon/Castanet plaideraient en faveur du contraire. Lors de ses fouilles à l'abri Blanchard, M. Castanet avait en effet remarqué la disparition des dépôts archéologiques vers le sud, bien avant la limite cadastrale qui délimite les deux abris. Des sondages dans la partie sud du terrain loué par L. Didon n'auraient rien livré. Précisons cependant que la falaise dans la partie sud de la parcelle Blanchard porte encore les traces d'aménagements troglodytiques (étude de Z. Garrett, en cours). En contrebas de cette structure, au bord du chemin, un sondage en 2008 a en outre livré des pierres à anneaux accompagnées de céramiques médiévales. On ne peut donc pas totalement exclure la possibilité d'une continuité entre les dépôts des abris Blanchard et Castanet à l'époque aurignacienne, continuité qui aurait été interrompue par une ou plusieurs vidanges post-paléolithiques.
Nos recherches sur l'abri Blanchard ont mis en lumière le caractère encore très lacunaire de nos connaissances sur l'Aurignacien récent. En dépit d'une plus forte attention ces dernières années (Chiotti 2000, 2003, 2005 ; Lucas 2000 ; Pesesse et Michel 2006 ; Michel 2010), l'Aurignacien récent reste le parent pauvre, les débats entourant l'émergence du Paléolithique supérieur en Europe ayant focalisé l'attention des chercheurs sur les industries des premières phases du techno-complexe.

Du point de vue de l'industrie lithique, il reste relativement mal connu. Les niveaux les plus répandus (présents dans toute l'Europe) correspondent à l'Aurignacien II de D. Peyrony (1934), avec deux faciès différents qui semblent avoir une valeur chronologique : un faciès à grattoirs à museau dominants et un faciès à burins busqués dominants (Aurignaciens Ila et IIr de F. Djindjian (1993)). En revanche, la fin de l'Aurignacien est plus problématique, les Aurignaciens III et IV de D. Peyrony restant assez mal définis et ne se retrouvant que rarement sur d'autres sites que La Ferrassie (Rigaud 1989). Ces industries de la fin de l'Aurignacien ont fait l'objet de définitions diverses et parfois contradictoires au cours du temps (Peyrony 1934 ; Sonneville-Bordes 1960 ; Delporte 1984 ; Rigaud 1989 ; Djindjian 1993 ; Demars 1994 ; Djindjian, Koslowski, Otte 1999 ; pour un essai de synthèse, voir Chiotti 2005). Plus récemment, est venu se rajouter un nouveau découpage de l'Aurignacien récent proposé par A. Michel (2010) qui complexifie encore la situation en redéfinissant certaines phases sous des appellations déjà existantes. Bien que parfois désignés sous des appellations différentes (Demars 1994 ; Michel 2010), la présence de deux faciès différents de l'Aurignacien II, dont la valeur chronologique semble acquise fait à peu près consensus. En revanche, les faciès postérieurs caractérisés principalement par la présence de burins des Vachons (Pesesse et Michel 2006) sont moins bien définis et font encore débat.

Pour ce qui concerne les productions en matières osseuses, on ne connaît encore que les pointes à base massive. Si leur valeur de fossile directeur de l'Aurignacien récent sensu lato reste valide, un important flou terminologique les entoure. Leurs attributs fonctionnels ne permettent pas toujours de les rattacher au registre des pointes de projectile et leurs caractéristiques morphologiques n'apparaissent pas suffisamment stables pour servir de cadre à une chronologie de la fin de l'Aurignacien. II est nécessaire de reprendre l'étude des séries pour tenter de mieux caractériser cette industrie. La tâche s'annonce toutefois difficile. En France, seule une vingtaine de sites (principalement en Dordogne) a livré des productions en matières osseuses rapportées à l'Aurignacien récent et souvent en faible quantité (Castanet, la Ferrassie, le Facteur, le Flageolet I, Cellier, etc.). Dans leur grande majorité, ils comprennent en outre plusieurs couches aurignaciennes et ont été fouillés à des dates anciennes. L'étude taphonomique de plusieurs d'entre eux a ainsi révélé des mélanges stratigraphiques illustrés par le caractère mixte de l'industrie lithique au sein des niveaux et/ou des remontages entre couches distinctes (ex: la Rochette, le Trou de la Chèvre, Roc de Combe, cf. Michel 2010 pour une synthèse). Dès lors, il apparaît indispensable d'entreprendre de nouvelles fouilles afin de baser notre réflexion sur des 
séquences archéo-stratigraphiques solides. C'est uniquement à ce prix qu'il nous sera possible de caractériser précisément les industries post-anciennes, de reconstituer la chronologie interne de l'Aurignacien du sud-ouest français et de suivre l'évolution des mutations technologiques et socioéconomiques amorcées depuis les phases anciennes du techno-complexe.

\section{Remerciements}

Les recherches menées dans le vallon de Castelmerle ont été soutenues depuis 1994 par un certain nombre d'organismes que nous souhaitons remercier ici : la United States National Science Foundation, la Direction régionale des affaires culturelles d'Aquitaine (DRAC-Aquitaine), la L.S.B. Leakey Foundation, la Reed Foundation, la Rock Foundation, la Fine Foundation, I'UMI 3199-CNRS-NYU (Center for International Research in the Humanities and Social Sciences), l'Institute for Ice Age Studies, la Theodore Dubin Foundation, le Service archéologique départemental de la Dordogne, la New York University, la Fondation Fyssen et la Fulbright Foundation. L'essentiel de ces recherches (et notamment l'étude des collections conservées aux États-Unis) a été effectué dans le cadre du programme de recherche franco-américain " Aurignacian Genius : Art, daily life and social identity of the first modern humans of Europe », UMI 3199-CNRS-NYU \& UMR 5608TRACES, financées par le Partner University Fund et la Andrew Mellon Foundation.

Nous remercions les conservateurs et personnels des musées américains qui nous ont accueillis et facilité l'accès aux collections : Paul Beelitz de l'American Museum of Natural History (New York), William A. Parkinson, Jamie Kelly et Ryan Gross du Field Museum of Natural History (Chicago), William Green et Nicolette Meister du Logan Museum of Anthropology (Beloit) ainsi que Patricia Hutchins et Abby du Wilson Museum (Castine).

Nos plus vifs remerciements reviennent aux membres de l'équipe dirigée par R. White qui conduisent depuis 2005 les recherches dans le vallon de Castelmerle et sans lesquels cet article n'aurait pu voir le jour.

\section{Références bibliographiques}

AVERBOUH, A., BUISSON D. 1996 - Approche morphofonctionnelle des objets nommés "lissoirs 》: essai d'établissement d'une fiche analytique théorique. Antiquités Nationales, 28, p. 41-46.

BOURRILLON R., WHITE R., TARTAR E., MENSAN R., CLARK A., CHIOTTI L., CASTEL J.-C., CRETIN C., HIGHAM T., RANLETT S., SISK M. sous presse - A new discovery of Aurignacian art at Abri Blanchard (Dordogne): implications for understanding Aurignacian graphic expression in Western and Central Europe. Journal of Human Evolution.

CHIOTTI L. 2000 - Lamelles Dufour et grattoirs aurignaciens (carénés et à museau) de la couche 8 de l'abri Pataud, Les Eyzies-de-Tayac, Dordogne. L'Anthropologie, 104,2 , p. 239-263.
CHIOTTI L. 2003 - Les productions lamellaires dans l'Aurignacien de l'abri Pataud, Les Eyzies-de-Tayac, Dordogne, France. Gallia Préhistoire, 45, p. 113-156.

CHIOTTI L. 2005 - Les industries lithiques aurignaciennes de l'abri Pataud, Dordogne, France. Les fouilles de Hallam L. Movius Jr. Oxford, Archaeopress, 349 p. (BAR International Series, 1392).

CHIOTTI L., CRETIN C. 2011 - Les mises en forme de grattoirs carénés / nucléus de l'aurignacien ancien de l'abri Castanet (Sergeac, Dordogne). paléo, 22, p. 69-84.

CHIOTTI L., CRETIN C., MORALA A. sous presse - Les industries lithiques des abris Blanchard et Castanet (Dordogne, France) : Nouvelles données issues des fouilles 2005-2012. In : R. White, R. Bourrillon (Ed.), Aurignacian Genius: Art, Technology and Society of the First Modern Humans in Europe. Symposium international de New York (8-10 Avril 2013). Toulouse, Palethnologie, (Palethnologie, 6).

DAVID N. C. 1966 - The Perigordian Vc: an Upper Palaeolithic Culture in Western Europe. Cambridge (MA) : Harvard University, 1966. 755 p., 84 fig., 83 pl. Ph. D. Dissertation N.D.

DELAGE F. 1938 - Abri de la Souquette. Bulletin de la Société Historique et Archéologique du Périgord, 65, p. 104-126.

DELLUC B., DELLUC G. 1978 - Les manifestations graphiques aurignaciennes sur support rocheux des environs des Eyzies (Dordogne). Gallia Préhistoire, 21, p. 213-438.

DELLUC B., DELLUC G. 1981 - La dispersion des objets de l'abri Blanchard (Sergeac, Dordogne). Bulletin de la Société d'Etudes et de Recherches Préhistoriques des Eyzies, 30, p. 77-95.

DELPORTE H. 1984 - L'Aurignacien de La Ferrassie. In : Delporte H., Le grand abri de La Ferrassie, fouilles 19681973. Marseille Université de Provence, p. 145-234 (Études quaternaires, 7).

DELPORTE H. 1991 - La séquence aurignacienne et périgordienne sur la base des travaux récents réalisés en Périgord. Bulletin de la Société Préhistorique Française, 88, p. 243-256.

DEMARS P.-Y. 1994 - L'économie du silex au Paléolithique supérieur dans le nord de l'Aquitaine. Analyse, synthèse et interprétations. Thèse de doctorat d'état, Université de Bordeaux 1, 2 volumes, 549 p. et $270 \mathrm{p}$.

DIDON L. 1911 - L'Abri Blanchard des Roches. Bulletin de la Société Historique et Archéologique du Périgord, 87, p. 246-241 et 321-345.

DJINDJIAN F. 1993 - L'Aurignacien du Périgord : une révision. Préhistoire européenne, 3, p. 29-54. 
DJINDJIAN F., KOSLOWSKI J., OTTE M. 1999 - Le Paléolithique supérieur en Europe. Paris : éditions Armand Colin, $474 \mathrm{p}$.

GOUTAS N. 2008 - Les pointes d'Isturitz sont-elles toutes des pointes de projectile ? Gallia Préhistoire, 50, p. 45-101.

GOUTAS N. 2009 - Réflexions sur une innovation technique gravettienne importante: le double rainurage longitudinal. Bulletin de la Société préhistorique française 106, 437-456.

GOUTAS N. 2013 - Nouvelles données sur l'industrie osseuse des grottes du Renne et du Trilobite à Arcy-surCure (Yonne, France) : vers l'identification de nouveaux marqueurs techniques et culturels du Gravettien moyen à burins du Raysse. In : P. Bodu, L. Chehmana, L. Klaric, L. Mevel, S. Soriano et N. Teyssandier (Ed.), Le Paléolithique supérieur ancien de l'Europe du Nord-Ouest : Réflexions et synthèses à partir d'un projet collectif de recherche sur le centre et le sud du Bassin parisien. Actes du colloque de Sens (15-18 avril 2009). Paris : Société préhistorique française, p. 89-116, (Mémoire de la Société préhistorique française, $\mathrm{LVI}$ ).

HENRI-MARTIN L. 1931 - La station aurignacienne de La Quina. Angoulême : Impr. Ouvrière.

KNECHT H. 1991 - Technological innovation and design during the Early Upper Paleolithic New York : New York University. Ph. D. Dissertation N.D.

KNECHT H. 1993 - Splits and Wedges: the techniques and technology of Early Aurignacian antler working. In : H. Knecht, A. Pike-Tay et R. White (Ed.), Before Lascaux : the complex record of the Early Upper Paleolithic, Boca Raton : CRC Press, p. 137-162.

KNECHT H. 1997 - Projectile points of bone, antler, and stone. Experimental explorations of manufacture and use. In : Knecht H. (Ed.), Projectile technology, Interdisciplinary contributions to archaeology. New York : Plenum Press, p. $192-212$

LEROY-PROST C. 1975 - L'industrie osseuse aurignacienne. Essai régional de classification : Poitou, Charente, Périgord. Gallia préhistoire, 18, p. 65-156.

LEROY-PROST C. 1979 - L'industrie osseuse aurignacienne. Essai régional de classification : Poitou, Charente, Périgord (suite). Gallia préhistoire, 22, p. 205-370.

LIOLIOS D. 1999 - Variabilité et caractéristiques du travail des matières osseuses au début de l'Aurignacien: Approche technologique et économique. Nanterre : Université Paris X Nanterre, 1999, 359 p. Thèse N.D.

LIOLIOS D. 2006 - Reflections on the role of bone tools in the definition of the Early Aurignacian. In : O. Bar-Yosef, Zilhão, J. (Eds.), Towards a definition of the Aurignacian, proceedings of the symposium held in Lisbon, Portugal, June 25-30, 2002. Lisbonne : Institut Portugais d'Archéologie, p. 37-52 (Trabalhos de arqueologia, 45).
LUCAS G. 2000 - Les industries lithiques du Flageolet I (Dordogne) : approche économique, technologique, fonctionnelle et analyse spatiale. Bordeaux : Université de Bordeaux 1, 2000, 2 vol., 307 p et 295 p. Thèse N.D.

MAC CURDY G.G. 1914 - Paleolithic art as represented in the collections of the American museum of New York. The American Museum Journal, 14, p. 225-237.

MICHEL A. 2010 - L'Aurignacien récent (post-ancien) dans le Sud-Ouest de la France : variabilité des productions lithiques. Révision taphonomique et techno-économique des sites de Caminade-Est, abri Pataud, Roc-de-Combe, Le Flageolet I, La Ferrassie et Combemenue. Bordeaux : Université de Bordeaux 1, 2010, 600p. Thèse N.D.

MOVIUS H. L. 1973 - Quelques commentaires supplémentaires sur les sagaies d'Isturitz: données de l'abri Pataud, Les Eyzies (Dordogne). Bulletin de la Société préhistorique française, 70, 3, p. 85-89.

PATOU-MATHIS M. dir. 2002 - Fiches typologiques de l'industrie osseuse préhistorique. Commission de nomenclature sur l'industrie de l'os préhistorique. Cahier $\mathrm{X}$ : Os à impressions et éraillures, Treignes : CEDARC, 136 p.

PELEGRIN J., O'FARELL M. 2005 - Les lamelles retouchées ou utilisées de Castanet. In : F. Le Brun-Ricalens, (Ed.), Productions lamellaires attribuées à l'Aurignacien : Chaînes opératoires et perspectives technoculturelles. $\mathrm{XIV}^{\circ}$ congrès de I'UISPP, Liège (2-8 Septembre 2001). Luxembourg : MNHA, p. 103-121 (ArcheoLogiques, 1).

PESESSE D., MICHEL A. 2006 - Le burin des Vachons : apports d'une relecture technologique à la compréhension de l'Aurignacien récent du Sud-Ouest de la France. Paleo, 18, p. 143-160.

PEYRONY D. 1928 - Pièces à languette de l'Aurignacien moyen. Anthropologie et Archéologie. Association Française pour l'avancée des sciences, p. 439-441.

PEYRONY D. 1933 - Les industries «Aurignaciennes» dans le bassin de la Vézère. Bulletin de la Société préhistorique française, 30, p. 543-559.

PEYRONY D. 1934 - La Ferrassie, Moustérien - Périgordien - Aurignacien. Préhistoire, t. III, p. 1-92.

PEYRONY D. 1935 - Le gisement de Castanet, vallon de Castelmerle, commune de Sergeac (Dordogne). Bulletin de la Société préhistorique française, 32, p. 418-443.

RIGAUD J.-Ph. 1989 - Le Paléolithique supérieur ancien en Aquitaine. In : J.-P. Mohen (Dir.), Le Temps de la Préhistoire, t. 1. Paris : Société préhistorique française, éditions Archéologia, p. 269-273.

SONNEVILLE-BORDES (de) D. 1960 - Le Paléolithique supérieur en Périgord. Bordeaux : Delmas, 558 p. 
TARTAR E. 2009 - De l'Os à l'Outil : Caractérisation technique, économique et sociale de l'utilisation de l'os à l'Aurignacien ancien. Étude de trois sites : l'Abri Castanet (secteurs nord et sud), Brassempouy (Grotte des Hyènes et Abri Dubalen) et Gatzarria. Paris : Université de Paris IPanthéon-Sorbonne, 2009, 2 t., 300 p. (t.1). Thèse N.D.

TARTAR E. 2012 - Réflexion autour de la fonction des retouchoirs en os de l'Aurignacien ancien. Bulletin de la Société préhistorique française, 109, p. 69-83.

TARTAR E. sous presse - Aurignacian osseous technology: a review of past beliefs and curent knowledge (with data from French sites). In : R. White, R. Bourrillon et F. Bon (Ed.), Aurignacian Genius: Art, Technology and Society of the First Modern Humans in Europe. Symposium international de New York (8-10 Avril 2013). Toulouse : Palethnologie, (Palethnologie, 6).

TARTAR E., WHITE R. 2013 - The manufacture of aurignacian split-based points: an experimental challenge. Journal of Archaeological Science, 40, p. 2723-2745.

TEJERO J.-M. 2013 - La explotación de las materias óseas en el Auriñaciense. Caracterización tecnoeconómica de las producciones del Paleolítico superior inicial en la Península Ibérica. Oxford: Archaeopress, 252 p.

TEXIER J.-P. 2009 - Histoire géologique de sites préhistoriques classiques du Périgord : une vision actualisée. La Micoque, la grotte Vaufrey, Le Pech de l'Azé I et II, La Ferrassie, l'abri Castanet, Le Flageolet, Laugerie Haute. Editions du CTHS, 25, 193 p.
WHITE R. 1992 - Bone, Antler and Ivory Objects from Abri Blanchard at the Logan Museum of Anthropology. In : R. White et L. B. Breitborde (Ed.), French Palaeolithic Collections in the Logan Museum of Anthropology, p. 97120, (Logan Museum Bulletin, vol. 1, N2).

WHITE R. 2002 - The historic legal context of foreign acqusitions of Paleolithic artifacts from the prerigord: 1900 to 1941. In: L.G. Straus (Ed.), The role of american archaeologists in the study of the European Upper Paleolithic (Oxford: Archaeopress), p. 71-83.

WHITE R. 2006a - L'affaire de l'abri du poisson : patrie et préhistoire, Périgueux : Editions Fanlac, $237 \mathrm{p}$.

WHITE R. (dir.) 2006b - Abri Castanet, secteur sud (commune de Sergeac, Dordogne). Rapport de fouille programmée. Année 2006. Rapport remis au SRA Aquitaine, $184 \mathrm{p}$.

WHITE R. 2006c - From Puente Viesgo to Central Park West: Hugo Obermaier, Nels Nelson and the American Museum of Natural History's collections from Cantabrian Spain. In: J.M. Maillo, E. Baquedano (Ed.), Miscelanea en homenaje a Victoria Cabrera. Cabrera. Alcala de Henares : Museo Arqueologico Regional, UNED, p. 58-77 (Zona Arqueologica 7, vol. 1).

WHITE R. (dir.) 2007 - Abri Castanet, secteurs sud et nord (commune de Sergeac, Dordogne). Rapport de fouille programmée. Année 2007. Rapport remis au SRA Aquitaine, $186 \mathrm{p}$. 


\section{ANNEXE}

Tableau 3 - Listing de l'industrie en matières osseuses de l'abri Blanchard conservée aux États-Unis (certaines pièces, exposées en vitrine à l'AMNH, n'ont pas pu être mesurées).

Table 3 - Listing of Blanchard osseous industry housed in US museums (dimensions are missing for some AMNH artifacts on exhibition).

\begin{tabular}{|c|c|c|c|c|c|c|c|c|}
\hline $\mathbf{N}^{\circ}$ & Pièce & Collection & $\begin{array}{c}\mathrm{N}^{\circ} \text { d'inventaire } \\
\text { musée }\end{array}$ & $\begin{array}{c}\text { Matière } \\
\text { première }\end{array}$ & $L$ & $I$ & e & $\begin{array}{l}\text { Illustrations } \\
\text { (Didon 1911) }\end{array}$ \\
\hline 1 & Pointe à base fendue & AMNH & $75.0 / 555$ & $\mathrm{BDC}$ & $>108$ & 21 & 9 & \\
\hline 2 & " & AMNH & $75.0 / 555$ & $\mathrm{BDC}$ & $>45$ & 9 & 8 & \\
\hline 3 & " & AMNH & $75.0 / 555$ & $\mathrm{BDC}$ & $>71$ & 14 & 6 & \\
\hline 4 & $"$ & AMNH & $75.0 / 555$ & BDC & $>50$ & 10 & 5 & \\
\hline 5 & $"$ & AMNH & $75.0 / 555$ & $\mathrm{BDC}$ & $>75$ & 9 & 6 & \\
\hline 6 & " & AMNH & $75.0 / 555$ & $\mathrm{BDC}$ & 110 & 14 & 6 & \\
\hline 7 & $"$ & AMNH & $75.0 / 555$ & $\mathrm{BDC}$ & $>77$ & 23 & 7 & \\
\hline 8 & " & AMNH & $75.0 / 555$ & $\mathrm{BDC}$ & $>67$ & 16 & 6 & \\
\hline 9 & $"$ & AMNH & $75.0 / 555$ & $\mathrm{BDC}$ & $>91$ & 15 & 6 & \\
\hline 10 & " & AMNH & $75.0 / 555$ & BDC & - & - & - & planche II, $\mathrm{n}^{\circ} 1$ \\
\hline 11 & " & AMNH & $75.0 / 555$ & BDC & - & - & - & \\
\hline 12 & " & AMNH & $75.0 / 555$ & BDC & - & - & - & \\
\hline 13 & " & AMNH & $75.0 / 555$ & BDC & - & - & - & \\
\hline 14 & " & AMNH & $75.0 / 555$ & $\mathrm{BDC}$ & $>123$ & 13 & 7 & \\
\hline 15 & $"$ & AMNH & $75.0 / 557$ & $\mathrm{BDC}$ & $>135$ & 18 & 7 & \\
\hline 16 & " & FM & 197838 & $\mathrm{BDC}$ & $>90$ & $>13$ & $>8$ & \\
\hline 17 & " & FM & 197840 & $\mathrm{BDC}$ & $>61$ & 13 & 6 & \\
\hline 18 & $"$ & FM & 219541 & $\mathrm{BDC}$ & $>49$ & 15 & 5 & planche II, $\mathrm{n}^{\circ} 3$ \\
\hline 19 & " & LMA & 4.05 .399 & $\mathrm{BDC}$ & $>99$ & 14 & 8 & \\
\hline 20 & $"$ & LMA & 4.05 .406 & $\mathrm{BDC}$ & $>73$ & 26 & 15 & \\
\hline 21 & $"$ & WM & w10012 & BDC & $>97$ & 19 & 7 & \\
\hline 22 & " & WM & w10023 & $\mathrm{BDC}$ & $>90$ & 13 & 7 & \\
\hline 23 & $"$ & WM & w11622 & BDC & 111 & 17 & 6 & \\
\hline 24 & Pointe à base massive & AMNH & $75.0 / 553$ & BDC & $>192$ & 30 & 11 & \\
\hline 25 & " & WM & w11629 & $\mathrm{BDC}$ & $>125$ & 13 & 9 & \\
\hline 26 & Pointe d'Isturitz (fragment) & LMA & 4.05 .390 & $\mathrm{BDC}$ & $>49$ & $>23$ & $>11$ & \\
\hline 27 & Pointe (fragment) & AMNH & $75.0 / 555$ & $\mathrm{BDC}$ & $>72$ & $>9$ & $>5$ & \\
\hline 28 & " & AMNH & $75.0 / 556$ & $\mathrm{BDC}$ & $>63$ & $>8$ & $>6$ & \\
\hline 29 & " & AMNH & $75.0 / 556$ & $\mathrm{BDC}$ & $>45$ & $>6$ & $>5$ & \\
\hline 30 & " & AMNH & $75.0 / 557$ & $\mathrm{BDC}$ & $>72$ & $>24$ & $>12$ & \\
\hline 31 & $"$ & AMNH & $75.0 / 557$ & $\mathrm{BDC}$ & $>156$ & 21 & $>11$ & \\
\hline 32 & " & AMNH & $75.0 / 557$ & BDC & - & - & - & \\
\hline 33 & " & FM & 197835 & $\mathrm{BDC}$ & $>112$ & $>7$ & $>7$ & \\
\hline 34 & $"$ & FM & 197842 & $\mathrm{BDC}$ & $>44$ & $>15$ & $>8$ & \\
\hline 35 & " & FM & 197843 & $\mathrm{BDC}$ & $>37$ & $>7$ & $>5$ & \\
\hline 36 & " & LMA & 4.05 .391 & $\mathrm{BDC}$ & $>22$ & $>10$ & $>5$ & \\
\hline 37 & $"$ & LMA & 4.05 .396 & $\mathrm{BDC}$ & $>36$ & $>7$ & $>3$ & \\
\hline 38 & " & WM & w10008 & $\mathrm{BDC}$ & $>75$ & 19 & $>8$ & \\
\hline 39 & $"$ & WM & w10071 & $\mathrm{BDC}$ & $>87$ & 23 & $>9$ & \\
\hline 40 & $"$ & WM & w11587 & BDC & $>84$ & $>10$ & $>7$ & \\
\hline 41 & $"$ & WM & w11636 & $\mathrm{BDC}$ & $>74$ & $>13$ & $>6$ & \\
\hline 42 & $"$ & WM & w11640 & $\mathrm{BDC}$ & $>63$ & $>9$ & $>4$ & \\
\hline 43 & Poinçon & AMNH & $75.0 / 556$ & OS & 70 & 7 & 4 & \\
\hline 44 & $"$ & AMNH & $75.0 / 556$ & OS & $>50$ & 12 & 5 & \\
\hline 45 & $"$ & AMNH & $75.0 / 556$ & os & $>57$ & 8 & 5 & \\
\hline 46 & " & AMNH & $75.0 / 556$ & OS & $>62$ & 6 & 7 & \\
\hline 47 & " & AMNH & $75.0 / 556$ & OS & $>63$ & $>5$ & $>5$ & \\
\hline 48 & $"$ & AMNH & $75.0 / 556$ & OS & $>64$ & $>4$ & $>4$ & \\
\hline 49 & " & AMNH & $75.0 / 556$ & OS & $>65$ & $>6$ & $>5$ & \\
\hline 50 & $"$ & AMNH & $75.0 / 556$ & OS & - & - & - & \\
\hline 51 & " & AMNH & 75.0 / 557 & OS & $>67$ & $>12$ & 4 & \\
\hline 52 & $"$ & FM & 197834 & OS & $>114$ & $>21$ & 10 & \\
\hline 53 & $"$ & LMA & 4.05 .388 & OS & $>36$ & $>8$ & $>3$ & \\
\hline 54 & " & LMA & 4.05 .392 & OS & $>25$ & $>6$ & $>5$ & \\
\hline 55 & $"$ & LMA & 4.05 .393 & OS & $>48$ & $>5$ & $>4$ & \\
\hline 56 & $"$ & LMA & 4.05 .394 & OS & $>15$ & $>2$ & $>2$ & \\
\hline 57 & " & LMA & 4.05 .395 & os & $>34$ & $>4$ & $>4$ & \\
\hline 58 & $"$ & LMA & 4.05 .400 & os & $>50$ & 11 & 9 & \\
\hline 59 & " & LMA & 4.05 .401 & OS & $>62$ & 9 & 6 & \\
\hline 60 & " & WM & w10003 & OS & $>65$ & 10 & 5 & \\
\hline 61 & " & WM & w10031 & os & $>49$ & 8 & 7 & \\
\hline 62 & $"$ & WM & w10072 & OS & $>62$ & 15 & 4 & \\
\hline
\end{tabular}




\begin{tabular}{|c|c|c|c|c|c|c|c|c|}
\hline $\mathbf{N}^{\circ}$ & Pièce & Collection & $\mathrm{N}^{\circ}$ d'inventaire & $\begin{array}{c}\text { Matière } \\
\text { première }\end{array}$ & $\mathbf{L}$ & I & e & $\begin{array}{l}\text { Illustrations } \\
\text { (Didon 1911) }\end{array}$ \\
\hline 63 & Bâtonnet & LMA & 4.05 .373 & $\mathrm{BDC}$ & $>30$ & 7 & 6 & \\
\hline 64 & $"$ & LMA & 4.05 .383 & OS/BDC & $>11$ & $>5$ & $>3$ & \\
\hline 65 & " & LMA & 4.05 .386 & $\mathrm{BDC}$ & $>23$ & 5 & 4 & \\
\hline 66 & Tube & LMA & 4.05 .372 & OS & $>21$ & $>8$ & $>2$ & \\
\hline 67 & $"$ & LMA & 4.05 .374 & OS & $>18$ & $>7$ & $>3$ & \\
\hline 68 & Lissoir & AMNH & $75.0 / 537$ & OS & $>225$ & 23 & 8 & planche $\mathrm{V}, \mathrm{n}^{\circ} 6$ \\
\hline 69 & $"$ & AMNH & $75.0 / 540$ & os & $>78$ & 14 & 5 & planche $\mathrm{VI}, \mathrm{n}^{\circ} 44$ \\
\hline 70 & $"$ & AMNH & $75.0 / 553+75.0 / 557$ & OS & $>218$ & 20 & 9 & \\
\hline 71 & $"$ & AMNH & $75.0 / 554$ & OS & $>52$ & $>15$ & $>4$ & \\
\hline 72 & $"$ & AMNH & $75.0 / 554$ & os & $>48$ & $>10$ & $>6$ & \\
\hline 73 & $"$ & AMNH & $75.0 / 554$ & os & $>37$ & $>13$ & $>6$ & \\
\hline 74 & $"$ & FM & $197841+197852$ & os & $>179$ & 17 & 5 & \\
\hline 75 & $"$ & LMA & 4.05 .389 & os & $>32$ & $>7$ & $>4$ & \\
\hline 76 & $"$ & WM & w10007 & os & 148 & 18 & 3,5 & \\
\hline 77 & $"$ & WM & w10026 & os & $>64$ & 15 & 4 & \\
\hline 78 & $"$ & WM & w10073 & OS & $>94$ & 18 & 4 & \\
\hline 79 & " & WM & w10075 & OS & $>31$ & $>6$ & $>3$ & planche $\mathrm{VI}, \mathrm{n}^{\circ} 49$ \\
\hline 80 & $"$ & WM & w10076 & os & $>113$ & 13 & 4 & \\
\hline 81 & $"$ & WM & w10127 & OS & $>40$ & $>12$ & $>3$ & \\
\hline 82 & $"$ & WM & w10154 & OS & $>48$ & 16 & 5 & \\
\hline 83 & $"$ & WM & w11597 & os & $>37$ & $>8$ & $>5$ & planche VI, $n^{\circ} 48$ \\
\hline 84 & Objet à extrémité mousse & AMNH & $75.0 / 553$ & $\mathrm{BDC}$ & 156 & 38 & 40 & planche $V, n^{\circ} 2$ \\
\hline 85 & " & AMNH & $75.0 / 557$ & OS & 166 & 16 & 7 & \\
\hline 86 & $"$ & FM & 197832 & $\mathrm{BDC}$ & 151 & 22 & 10 & planche II, $n^{\circ} 18$ \\
\hline 87 & $"$ & FM & 197837 & $\mathrm{BDC}$ & $>101$ & $>12$ & 7 & \\
\hline 88 & Outil intermédiaire & AMNH & $75.0 / 558$ & os & $>110$ & 18 & 7 & \\
\hline 89 & " & LMA & 4.05 .397 & OS & $>112$ & $>17$ & $>7$ & \\
\hline 90 & Bâton percé & AMNH & $75.0 / 564$ & $\mathrm{BDC}$ & 166 & 95 & 45 & planche III, $n^{\circ} 2$ \\
\hline 91 & $"$ & FM & 197859 & BDC & $>52$ & $>83$ & $>20$ & \\
\hline 92 & Côte décorée & AMNH & $75.0 / 560$ & OS & - & - & - & \\
\hline 93 & $"$ & WM & w10150 & os & $>42$ & $>18$ & $>5$ & \\
\hline 94 & Retouchoir & $\mathrm{AMNH}$ & $75.0 / 538$ & os & 122 & 30 & 17 & \\
\hline 95 & " & AMNH & $75.0 / 552$ & os & 79 & 26 & 22 & \\
\hline 96 & Objet indéterminé & $\mathrm{AMNH}$ & $75.0 / 553$ & $\mathrm{BDC}$ & $>88$ & $>27$ & $>9$ & \\
\hline 97 & " & FM & 197856 & $\mathrm{BDC}$ & $>106$ & $>11$ & 7 & \\
\hline 98 & $"$ & LMA & 4.05 .404 & OS & $>98$ & 8 & 8 & \\
\hline 99 & Pièce en cours de mise en forme & AMNH & $75.0 / 568$ & os & 150 & 19 & 7 & \\
\hline 100 & Ebauche de pointe à base fendue & AMNH & $75.0 / 555$ & $\mathrm{BDC}$ & 81 & 11 & 5 & planche II, $n^{\circ} 5$ \\
\hline 101 & " & AMNH & $75.0 / 555$ & $\mathrm{BDC}$ & $>134$ & 22 & 9 & \\
\hline 102 & $"$ & AMNH & $75.0 / 557$ & $\mathrm{BDC}$ & $>164$ & $>28$ & 10 & \\
\hline 103 & $"$ & AMNH & $75.0 / 557$ & $\mathrm{BDC}$ & - & - & - & \\
\hline 104 & " & FM & 197836 & $\mathrm{BDC}$ & $>99$ & 14 & 6 & \\
\hline 105 & $"$ & FM & 197854 & $\mathrm{BDC}$ & $>80$ & 18 & 8 & \\
\hline 106 & " & WM & w10049 & $\mathrm{BDC}$ & $>96$ & 23 & 8 & \\
\hline 107 & Ebauche de pointe à base massive & $\mathrm{AMNH}$ & $75.0 / 557$ & $\mathrm{BDC}$ & - & - & - & \\
\hline 108 & Déchet de sectionnement & FM & 197855 & os & 77 & 16 & 13 & \\
\hline 109 & $"$ & WM & w10060 & OS & $>116$ & $>20$ & $>9$ & \\
\hline 110 & Pièce avec traces de sciage & FM & 197846 & os & 40 & 15 & 15 & \\
\hline 111 & " & FM & 197857 & OS & 85 & 7 & 7 & \\
\hline 112 & Pièce avec traces de rainurage & FM & 197839 & $\mathrm{BDC}$ & $>87$ & $>25$ & 9 & \\
\hline 113 & $"$ & LMA & 4.05 .403 & $\mathrm{BDC}$ & $>52$ & $>20$ & 7 & \\
\hline 114 & $"$ & WM & w10079 & os & 63 & 13 & 11 & \\
\hline 115 & Déchet de fente (pièce à languette) & AMNH & $75.0 / 559$ & $\mathrm{BDC}$ & 67 & 19 & 8 & \\
\hline 116 & $"$ & $\mathrm{AMNH}$ & $75.0 / 559$ & BDC & $>58$ & 26 & 12 & \\
\hline 117 & $"$ & AMNH & $75.0 / 559$ & $\mathrm{BDC}$ & 96 & 28 & 14 & \\
\hline 118 & " & FM & 197849 & $\mathrm{BDC}$ & $>54$ & 18 & 9 & \\
\hline 119 & Déchet de fente (pièce à languette) & FM & 197851 & $\mathrm{BDC}$ & $>71$ & $>21$ & $>10$ & \\
\hline 120 & " & FM & $197876-4$ & $\mathrm{BDC}$ & 16 & 19 & 6 & \\
\hline 121 & $"$ & LMA & 4.05 .405 & $\mathrm{BDC}$ & 49 & 19 & 7 & \\
\hline 122 & $"$ & WM & w10080 & $\mathrm{BDC}$ & 43 & 17 & 7 & \\
\hline 123 & Déchet de sectionnement & FM & 197850 & $\mathrm{BDC}$ & $>59$ & $>18$ & 7 & \\
\hline 124 & " & WM & w10027 & $\mathrm{BDC}$ & 58 & 19 & 8 & \\
\hline
\end{tabular}




\begin{tabular}{|c|c|c|c|c|c|c|c|c|}
\hline $\mathbf{N}^{\circ}$ & Pièce & Collection & $\mathbf{N}^{\circ}$ d'inventaire & $\begin{array}{c}\text { Matière } \\
\text { première }\end{array}$ & L & I & e & $\begin{array}{l}\text { Illustrations } \\
\text { (Didon 1911) }\end{array}$ \\
\hline $\mathbf{1 2 5}$ & Déchet de sectionnement & WM & w10078 & BDC & 67 & 18 & 8 & \\
$\mathbf{1 2 6}$ & Déchet de mise en forme & AMNH & $75.0 / 556$ & OS & 40 & 7 & 7 & \\
$\mathbf{1 2 7}$ & $"$ & AMNH & $75.0 / 556$ & BDC & 36 & 9 & 6 & \\
$\mathbf{1 2 8}$ & $"$ & AMNH & $75.0 / 556$ & BDC & 36 & 8 & 6 & \\
$\mathbf{1 2 9}$ & " & AMNH & $75.0 / 556$ & OS & $>30$ & $>12$ & 4 & \\
$\mathbf{1 3 0}$ & Base de bois avec traces d'entaillage & AMNH & $75.0 / 565$ & BDC & - & - & - & \\
\hline
\end{tabular}


